\title{
Residual Inhibition Functions Overlap Tinnitus Spectra and the Region of Auditory Threshold Shift
}

\author{
Larry E. Roberts, ${ }^{1}$ Graeme Moffat, ${ }^{1}$ Michael Baumann, ${ }^{2}$ Lawrence M. Ward, ${ }^{2,3}$ \\ AND DANIEL J. BOSNYAK ${ }^{1}$ \\ ${ }^{1}$ Department of Psychology, Neuroscience, and Behaviour, McMaster University, 1280 Main Street West, Hamilton, \\ ON, Canada L8S $4 K 1$ \\ ${ }^{2}$ Department of Psychology, University of British Columbia, Vancouver, British Columbia, Canada V6T 1Z4 \\ ${ }^{3}$ The Brain Research Centre, University of British Columbia, Vancouver, British Columbia, Canada V6T 1Z4
}

Received: 18 December 2007; Accepted: 1 August 2008; Online publication: 20 August 2008

\begin{abstract}
Animals exposed to noise trauma show augmented synchronous neural activity in tonotopically reorganized primary auditory cortex consequent on hearing loss. Diminished intracortical inhibition in the reorganized region appears to enable synchronous network activity that develops when deafferented neurons begin to respond to input via their lateral connections. In humans with tinnitus accompanied by hearing loss, this process may generate a phantom sound that is perceived in accordance with the location of the affected neurons in the cortical place map. The neural synchrony hypothesis predicts that tinnitus spectra, and heretofore unmeasured "residual inhibition functions" that relate residual tinnitus suppression to the center frequency of masking sounds, should cover the region of hearing loss in the audiogram. We confirmed these predictions in two independent cohorts totaling 90 tinnitus subjects, using computer-based tools designed to assess the psychoacoustic properties of tinnitus. Tinnitus spectra and residual inhibition functions for depth and duration increased with the amount of threshold shift over the region of hearing impairment. Residual inhibition depth was shallower when the masking sounds that were used to induce residual inhibition showed decreased correspondence with the frequency spectrum and bandwidth of the tinnitus. These findings suggest that tinnitus and
\end{abstract}

Correspondence to: Larry E. Roberts - Daniel J. Bosnyak · Department of Psychology, Neuroscience, and Behaviour · McMaster University · 1280 Main Street West, Hamilton, ON, Canada L8S 4K1. Telephone: +1-905-5259140; fax: +1-905-5296225; email: roberts@mcmaster.ca; email: bosnyak@mcmaster.ca its suppression in residual inhibition depend on processes that span the region of hearing impairment and not on mechanisms that enhance cortical representations for sound frequencies at the audiometric edge. Hearing thresholds measured in age-matched control subjects without tinnitus implicated hearing loss as a factor in tinnitus, although elevated thresholds alone were not sufficient to cause tinnitus.

Keywords: residual inhibition, tinnitus spectra, neural synchrony, cortical reorganization

\section{INTRODUCTION}

In his classic studies of tinnitus masking, Feldman (1971) observed that a substantial number of individuals suffering from tinnitus experienced a temporary reduction of their tinnitus following the cessation of masking sounds. This phenomenon has come to be called "residual inhibition" (RI) in the tinnitus literature, although "post-masking suppression" (adapted from Tyler et al. 1984) is more neutral with regard to its possible underlying mechanisms. It is known from foundation studies now more than 20 years old that the duration of RI is typically on the order of tens of seconds, although reports of RI lasting minutes or longer are not uncommon (Vernon and Meikle 2003). For RI to be experienced, masking sounds are presented at levels exceeding the loudness of tinnitus for at least $10 \mathrm{~s}$, with RI duration increasing as a negatively accelerated function of masking 
duration, and approaching asymptote around $1 \mathrm{~min}$ with little gain thereafter (Terry et al. 1983). It is noteworthy that, although RI is one of the few procedures known to reduce or eliminate tinnitus for brief periods, relationships of RI depth and duration to the psychoacoustic properties of tinnitus, the characteristics of masking sounds, and hearing loss have not been extensively explored (Henry and Meikle 2000). In this paper, we describe "RI functions" that relate RI to properties of masking stimuli and to the psychoacoustic properties of tinnitus using computerized tools designed for this purpose. It is possible (although by no means certain) that the mechanisms that underlie RI overlap those that generate tinnitus. Our goal was to better understand the mechanisms of tinnitus by identifying the conditions that foster its suppression in RI.

If RI and tinnitus share common mechanisms, studies of RI are best guided by advances in our knowledge of the pathophysiology of tinnitus. Although the specific role of hearing loss is debated (see Weisz et al. 2006), most cases of tinnitus are accompanied by hearing impairment associated with noise exposure or the aging process. One consequence of hearing loss identified by studies of animals exposed to noise trauma is a reorganization of the tonotopic map in primary auditory cortex (A1), such that sound frequencies near the edge of the cortical region of normal hearing come to be over-represented at the expense of sound frequencies in the region affected by hearing impairment (Robertson and Irvine 1989; Harrison et al. 1991; Rajan et al. 1993; Eggermont and Komiya 2000). It has been proposed that this overrepresentation of edge frequencies corresponds to the tinnitus sensation (see Rauschecker 1999, for a review). A second hypothesis proposed by Llinás et al. (2005) and others (e.g., Kiang et al. 1969) attributes tinnitus to a contrast enhancement of edge frequency percepts similar to that observed in other sensory systems at the borders of discontinuities of excitation and inhibition in sensory maps. Alternatively, tinnitus may be more closely related to changes in the dynamical activity of cortical neurons that occur not at the audiometric edge but rather throughout the cortical regions affected by hearing impairment (Eggermont and Roberts 2004). Animal studies have shown that, after noise-induced hearing loss, an increase occurs in the spontaneous firing rates of auditory cortical and subcortical neurons (although not in auditory nerve fibers) that is followed within hours by an increase in synchronous activity among neurons in the auditory cortex. Spontaneous firing rates increase for neurons inside as well as outside of the cortical tonotopic regions affected by hearing impairment, but enhanced neural synchrony (which may be forged by a usedependent mechanism) appears to be largely confined to and distributed across the damage-affected regions of the tonotopic map (Seki and Eggermont 2003; Noreña and Eggermont 2003). These neural changes may develop when feed-forward inhibition that normally restricts neural activity to sound frequencies contained in the stimulus (Cruikshank et al. 2007) is diminished after hearing injuries. Human functional brain imaging studies using the magnetically recorded $40-\mathrm{Hz}$ auditory steady-state response, which is generated in the region of the auditory core, have corroborated tonotopic map reorganization in human tinnitus sufferers as well as increased synchronous activity in this region that may correspond to the tinnitus percept (Wienbruch et al. 2006).

The experiment reported in this paper was guided by the hypothesis that increased synchronous activity occurring in regions of the auditory cortex affected by hearing loss is the principal neural correlate of tinnitus. This hypothesis has several implications for the psychoacoustic properties of tinnitus, for tinnitus masking, and for understanding RI. First, because the neural hypersynchrony believed to underlie tinnitus appears to be confined to tonotopic regions affected by hearing loss, tinnitus masking should be most effective for sounds that cover the hearing loss region. In his classic studies, Feldman categorized tinnitus masking effects into three main types according to whether auditory thresholds and tinnitus masking curves converged only at some frequencies ("convergence" type), at most frequencies ("congruence" type), or showed only a weak trend ("distance" type). The existence of these patterns was confirmed by subsequent studies using pure tones or narrow band noise as masking stimuli (Mitchell 1983; Tyler and Conrad-Ames 1984) with convergence tending to be the most common form $(53 \%$ in the study of Mitchell). However, when masker intensity is calculated as sensation level (SL) in these studies, it appears that for each masking type the sounds that best mask tinnitus are those with frequencies in the region where impairments of hearing are present (Roberts 2007). Almost all participants in these studies reported masking when presented with sounds in this region (94\% in the study of Mitchell 1983).

A second implication of the hypersynchrony hypothesis is that the tinnitus frequency region (the tinnitus "spectrum") should overlap the frequency range where hearing loss occurs. Although tinnitus percepts are often characterized in terms of a single dominant frequency (the tinnitus "pitch"), a limitation of this convention is that it may not accurately describe the bandwidth of the tinnitus even in narrowband (tonal) cases. Noreña et al. (2002) measured tinnitus frequencies by asking subjects to rate the degree to which each of the ten pure tones corresponded to a component of their tinnitus sensation. 
Each of the ten individuals tested (all reporting a tonal sound to their tinnitus) judged several frequencies to resemble their tinnitus sensation, and these frequencies were confined to and spanned the region of hearing impairment. Roberts et al. (2006) obtained similar results when tinnitus spectra were measured by an independent method resembling that of Noreña et al. (2002).

Finally, the hypersynchrony hypothesis implies a relationship between the tinnitus spectrum, hearing thresholds, and heretofore unmeasured "RI functions" that relate RI depth and duration to the frequency of the sounds used to induce RI. The study reported in this paper addressed this question. According to the neural synchrony hypothesis (Eggermont and Roberts 2004), when masking sounds are presented at supra-threshold levels covering the region of hearing impairment, feed-forward inhibition may be increased in the affected regions of the tonotopic map, disrupting the synchronous neural activity believed to underlie the tinnitus sensation. The result is tinnitus masking and, following cessation of the masker, a gradual recovery of tinnitus during a period of RI. Tinnitus recovery was proposed to reflect the resumption of synchronous activity in frequency regions of the auditory cortex in which inhibition was deficient owing to hearing impairment.

In the present study, we evaluated RI functions and tinnitus spectra in 90 tinnitus subjects, using stimuli with center frequencies covering the region of hearing impairment in the audiogram. We studied both bilateral and unilateral cases of tinnitus. Audiograms were measured either to $20 \mathrm{kHz}$ or to the limit of hearing. We predicted that optimal RI would be observed for masking sounds in the frequency range of the tinnitus spectrum and the region of hearing loss, provided that the masking sounds could be heard. We first describe three computer-based tools that were developed to measure tinnitus spectra and RI functions in order to assess these predictions. Baseline data are then reported and related to audiometric function for two cohorts of participants assessed by independent laboratories. Audiometric data were also gathered from 31 age-matched controls reporting no tinnitus in order to assess the role of hearing loss in tinnitus.

\section{METHODS}

\section{Subjects}

Two independent groups of tinnitus subjects were studied, one in Hamilton, Ontario, and the other in Vancouver, British Columbia. The Hamilton sample consisted of 74 adults (mean age, $58.6 \pm 1 \mathrm{SD}=$ 10.9 years; range, 22-81 years, 48 men) recruited from the ENT clinic at McMaster University Medical Center or by advertisements in the local newspaper. The Vancouver sample consisted of 16 adults (mean age, 59.1 \pm 12.6 years; range, $29-75$ years, 11 men) recruited from ENT clinics at hospitals affiliated with the University of British Columbia (UBC) or by newspaper advertisements. All subjects reported a chronic stable tinnitus persisting an average of 10.6 \pm 7.4 years (Hamilton) and $13.1 \pm 8.6$ years (Vancouver). Subjects gave a mean loudness rating for their tinnitus of $43.9 \pm 18.1$ (Hamilton) and 39.3 \pm 12.8 (Vancouver) corresponding to the midpoint between "moderate" and "strong" on a logarithmic Borg CR100 psychophysical scale known to relate subjective loudness to sound pressure level as a power function in normal hearing subjects (Borg and Borg 2001). Their mean total score on the Tinnitus Handicap Questionnaire (Kuk et al. 1990) was $32.0 \pm 18.9$ (Hamilton) and $31.5 \pm 20.0$ (Vancouver). A preliminary report of 24 cases of bilateral tinnitus from the Hamilton cohort has been described previously (Roberts et al. 2006).

An additional group of subjects reporting no history of tinnitus ( $n=31$ controls; mean age, 56.6 \pm 15.6 years, 17 men) was recruited in Hamilton and matched in age to the Hamilton tinnitus group. In a single session, control subjects completed the same structured interview given to tinnitus subjects and had their audiograms measured up to $20 \mathrm{kHz}$ or to the limit of hearing. Control subjects, however, did not complete the three computer-based tools described below for the measurement of tinnitus and RI. Comparisons of hearing function between the control and the tinnitus subjects were confined to the Hamilton sample where hearing function was measured with identical equipment.

Informed consent was obtained in accordance with procedures approved by ethics committees at McMaster University and the University of British Columbia. Subjects were reimbursed for their parking fees but were not otherwise remunerated.

\section{Apparatus and procedure}

Assessment tools for tinnitus subjects were three adaptive, subject-directed, computerized procedures called the Familiarization Program, Tinnitus Tester, and RI Tester. Each tool was programmed in Visual Basic running in Windows XP-Pro. All stimuli used in the experiment (with the exception of the "custom masker" described below) were prepared digitally in Matlab and saved as 48-kHz sampling rate, 16-bit pcm wave files at $6 \mathrm{~dB}$ below full scale. Stimuli consisted of pure tones and two sets of band-pass noise stimuli with 11 different center frequencies ranging from 0.5 to $12.0 \mathrm{kHz}$ but differing between sets in bandwidth. The two bandwidths were approximately $\pm 5 \%$ of the 
center frequency (CF) at $-10 \mathrm{~dB}$ (called BPN5 herein) and the other $\pm 15 \%$ of $\mathrm{CF}$ at $-10 \mathrm{~dB}$ (called BPN15 herein). The bandwidths of the BPN5 and BPN15 sounds were designated to represent "ringing" and "hissing" tinnitus, respectively (spectra are shown for CFs of 0.5 and $5.0 \mathrm{kHz}$ in Fig. 1B). The BPN15 stimuli were generated using second-order Chebychev type 1 digital bandpass filters. BPN5 stimuli were generated using a Gaussian-shaped FFT filter with FWHM at $0.05^{*} \mathrm{CF}$. At McMaster, sounds were delivered by a Creative Audigy2 sound card that was connected to a Tucker Davis PA5 attenuator and then to Sennheisser HDA-200 headphones through a Tucker Davis HB7 headphone buffer. All stimulus attenuation was done by the PA5 in order to preserve signal quality. The Windows volume controller was set at $70 \%$ maximum value and the headphone buffer to an attenuation of $15 \mathrm{~dB}$. This system delivered sounds up to $95 \mathrm{~dB}$ SPL from 0.5 to $12.0 \mathrm{kHz}$ (calibrated by a Bruel and Kjaer sound level meter coupled to the headphones, C scale). At UBC, sounds were generated by identical equipment but without a headphone buffer. The Windows volume controller was set at $100 \%$. It should be noted that while the maximum sound intensity accommodated by these systems was different, the sound intensities actually delivered to the subjects depended on the extent of their hearing impairment and their sound level matches in the Tinnitus and RI Testers. A nominal maximum sound level of $95 \mathrm{~dB}$ SPL was adopted at both sites, although this was exceeded by $\sim 5 \mathrm{~dB}$ for some subjects and stimuli in the Vancouver sample. Behavioral responses were recorded by a Powermate USB Multimedia controller (Griffin Technologies), which allowed subjects to turn and then depress a bidirectional dial to record their decisions.

In their first session, tinnitus subjects were given a structured interview to obtain information about their tinnitus and completed the Tinnitus Handicap Questionnaire. Audiograms were measured to the limit of

\section{A}

\section{Tinnitus Tester}

(1) Location of tinnitus (left, right or both ears)

(2) Adjust $500 \mathrm{~Hz}$ sound to comfortable level (This level used to present sounds in step 3 \& 4)

(3) Bandwidth of tinnitus (sound clips illustrating tonal, ringing, hissing tinnitus)

(4) Pulsating or continuous (two sound clips)

(5) Rate loudness of tinnitus on Borg100 Scale

(6) Adjust 11 sounds differing in CF to match loudness of tinnitus (The sounds are presented at the bandwidth chosen in Step 3) (Equates sounds for loudness and estimates the loudness of tinnitus)

(7) Rate 11 sounds for likeness to the pitch of tinnitus (Likeness ratings give the tinnitus spectrum)

(8) Subjects adjust three masking sounds to equal 1 kHz @65 dB SL (9) Brief test for residual inhibition (RI)

\section{C}

\section{RI Tester}

(1) Measure threshold of $1 \mathrm{kHz}$ tone

(2) Adjust the loudness of $1 \mathrm{WN}$ and 11 BPN15 maskers to equal the loudness of a $1 \mathrm{kHz}$ tone @ 65 dB SL

(3) Measure RI Function

Listen to tinnitus for 30 seconds

Listen to 1 of 11 BPN15 maskers or WN for $30 \mathrm{sec}$

Rate with dial whether tinnitus has changed

Press dial when tinnitus has recovered (45 sec limit)

After 30 seconds delay, procedure resumes

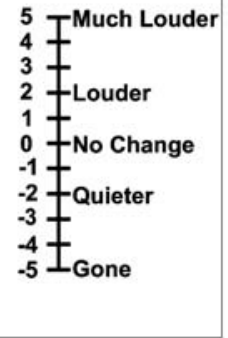

B
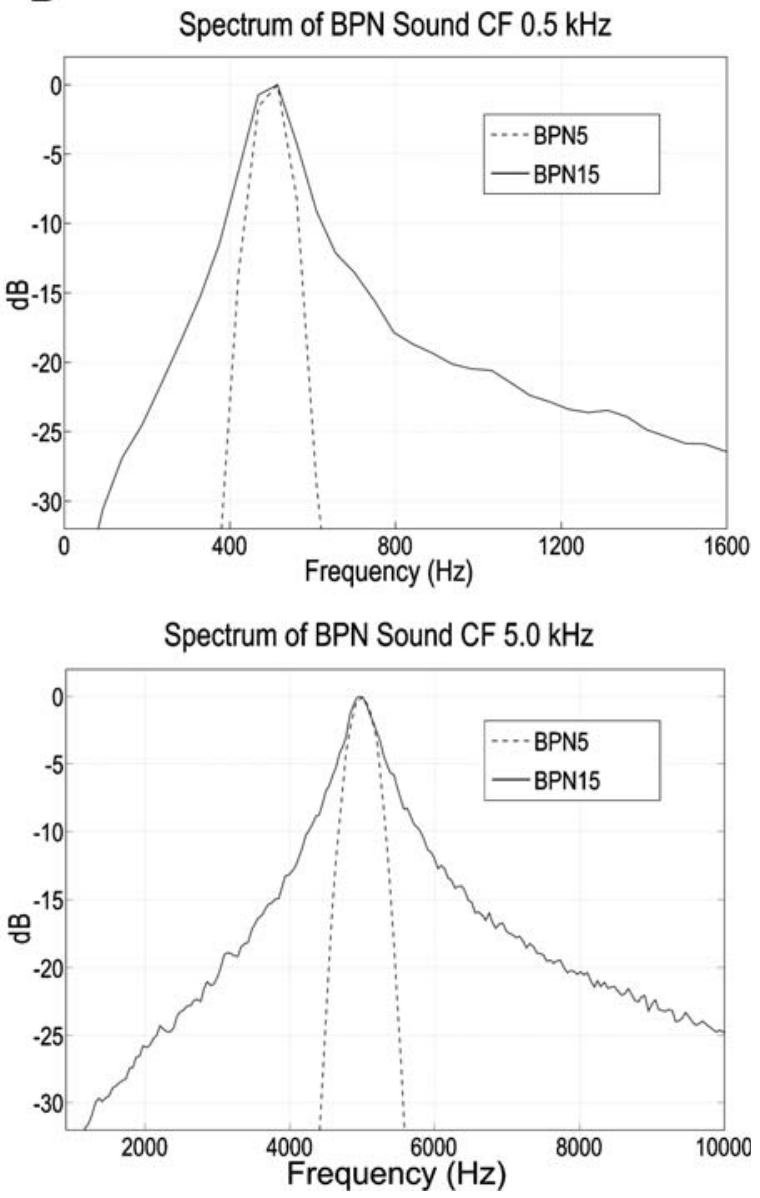

FIG. 1. Summary of steps in the Tinnitus Tester and residual inhibition tester. A The Tinnitus Tester. A familiarization program was administered before the Tinnitus Tester to acquaint subjects with the user interface and to illustrate the dimensions of pitch and loudness (see text for details). B Spectra of the BPN5 and BPN15 sounds at a CF of $0.5 \mathrm{kHz}$ (top) and $5.0 \mathrm{kHz}$ (bottom). The spectra for BPN5 and BPN15 sounds at other CFs were similarly shaped. C Residual inhibition tester. $W N$ white noise masker. 
hearing (20 kHz maximum) using a GSI-61 clinical audiometer (Hamilton sample) or a calibrated staircase procedure to $12 \mathrm{kHz}$ (Vancouver sample). Subjects completed the Familiarization Program and then the Tinnitus Tester, which assessed psychoacoustic properties of tinnitus. The session lasted about $2 \mathrm{~h}$. In a second session, 1-3 weeks later, the RI function was measured by the RI tester. A subset of subjects $(n=$ 17, see later) repeated the Tinnitus Tester approximately 1 week before the RI function was determined.

1. Familiarization Program. This program introduced subjects to the graphical user interface used for all procedures. By proceeding stepwise through a brief series of tasks, subjects learned how the computer program responded to their input from the dial by changing the sounds they heard and the images seen on the screen. The tasks also familiarized subjects with the concepts of loudness and pitch, which changed on separate trials as subjects manipulated dial settings. The Familiarization Program required about 15 min for completion.

2. Tinnitus Tester. This computerized procedure assessed the quality of the tinnitus sensation (ear, loudness, bandwidth, and frequency spectrum to limit of the subjects' audiogram) and gave a brief test for RI. The following steps were completed in the order indicated (see Fig. 1A). Step 1: Localize your tinnitus sensation. Subjects used the dial to select one of three options, left ear, right ear, or both ears. Step 2: adjustment of sound intensity. Subjects used the dial to adjust the loudness of a $0.5-\mathrm{kHz}$ pure tone to a comfortable level. This level was used in steps 3 and 4 to present sound clips for tinnitus assessment. Step 3: bandwidth of tinnitus. Subjects indicated whether their tinnitus was "tonal," "ringing," or "hissing." Three sound clips were presented to illustrate these choices, consisting of a $5-\mathrm{kHz}$ pure tone (tonal tinnitus), a BPN5 noise with $\mathrm{CF}=5.0 \mathrm{kHz}$ (ringing tinnitus), and a BPN15 noise with $\mathrm{CF}=5.0 \mathrm{kHz}$ (hissing tinnitus). Subjects used the dial to sample the sounds and indicate their choice. Step 4: temporal properties. Subjects indicated whether their tinnitus was steady or pulsing. Sound clips matched to the bandwidth chosen in the previous step were presented to illustrate their two choices. Step 5: tinnitus loudness rating. Subjects rated the perceived loudness of their tinnitus in the absence of external sound, using the dial to select a position on a Borg CR100 scale with the following quasilogarithmic anchors: 0 "extremely weak," 30 "moderate," 50 "strong," 70 "very strong," and 100 "extremely strong" (Borg and Borg 2001). Step 6: tinnitus loudness matching. Subjects were presented with sound clips with center frequencies ranging from 0.5 to $12.0 \mathrm{kHz}$. Bandwidth was determined by the selection made in step 3 (pure tone, BPN5, or BPN15). Subjects adjusted the loudness of each sound to match the loudness of their tinnitus, up to a maximum sound pressure level (SPL) of $95 \mathrm{~dB}$. Each sound was presented two times in a random order. Step 7: tinnitus likeness ratings. Subjects rated the pitch of each of the sounds presented in step 6 for similarity (likeness) to the pitch of their tinnitus using a Borg CR100 scale $(0=$ not at all, $30=$ not very similar, $50=$ somewhat similar, $70=$ very similar, and $100=$ identical). Each sound was presented three times in a random order. A profile of the tinnitus spectrum was thus generated. Step 8: sound threshold at $1.0 \mathrm{kHz}$. Subjects increased the loudness of a $1.0 \mathrm{kHz}$ tone until it was just audible. Sixty-five decibels was added to this level. Subjects then matched the loudness of each of three maskers to this 65-dB SL stimulus. Two masking stimuli were BPN15 noise maskers with center frequencies of 0.5 and $5.0 \mathrm{kHz}$. The third masking stimulus was a custom masker generated by the computer program according to the spectrum of the tinnitus measured in step 7. The custom masker for tonal and ringing tinnitus was the single pure tone or BPN5 sound, respectively, that was given the highest likeness rating. For hissing tinnitus, the custom masker was a combination of BPN15 sounds with a likeness rating of $\geq 40$ weighted in proportion to the mean likeness match given for each sound by the subject. Step 9. brief RI test. The three masking stimuli of step 8 were tested for RI. On trials of $60 \mathrm{~s}$ duration, subjects listened to their tinnitus for $30 \mathrm{~s}$ and then listened to a masker for $30 \mathrm{~s}$. After the masker subjects adjusted and then depressed the dial to indicate whether their tinnitus had increased ( +5 maximum), decreased $(-5$ meant tinnitus was eliminated), or had not changed (0). Ratings were given separately for each ear if bilateral tinnitus was indicated in step 1 . The next trial commenced after the rating(s) had been given. Each masking stimulus was tested three times by this procedure in a random order. The Tinnitus Tester required about $1 \mathrm{~h}$ for completion.

3. Residual inhibition tester. The RI tester measured "RI functions" that related the magnitude and duration of RI to a standardized set of 11 BPN15 maskers differing in $\mathrm{CF}$ and to a white noise (WN) masker. The stepwise progression of the RI tester was as follows (Fig. 1C). Step 1: threshold at $1.0 \mathrm{kHz}$. Using the dial, subjects completed a staircase procedure measuring the threshold of a $1.0-\mathrm{kHz}$ pure tone. Sixty-five decibels was added to this measurement for step 2. Step 2: loudness matching. Subjects adjusted the loudness of each of the 11 
BPN15 maskers and one $\mathrm{WN}$ masker to match the loudness of a 1-kHz tone presented at $65 \mathrm{~dB}$ SL, up to a limit of $\sim 95 \mathrm{~dB}$ SPL. The CFs of the BPN15 maskers were $0.5,1.0,2.0,3.0,4.0,5.0$ 6.0, 7.0, 8.0, 10.0 , and $12.0 \mathrm{kHz}$. Stimuli were presented once in a random order. The loudness levels determined by this step (which equated loudness over $\mathrm{CF}$ ) were used to present sounds in the next step. Step 3: RI function. Subjects listened to each of the 12 masking sounds (11 BPN15 stimuli and $1 \mathrm{WN}$ sound) three times on discrete trials given in a random order. On each trial (see Fig. 1C), subjects listened to their tinnitus for $30 \mathrm{~s}$ followed by a masker for $30 \mathrm{~s}$; an RI rating was then given using the same rating procedure of step 9 in the Tinnitus Tester. This procedure yielded a measure of RI depth (the rated decrease in the loudness of tinnitus sensation after the cessation of the masker). After the rating had been given, subjects depressed the dial a second time when their tinnitus had returned to normal. This procedure yielded a measure of RI duration (the time interval between the two dial presses, to a time-out limit of $45 \mathrm{~s})$. This estimate was conservative because it did not include the time required to rate RI depth. Following the second dial press or $45 \mathrm{~s}$ (whichever came first), the next trial commenced. The RI test required about $1 \mathrm{~h}$ for completion. ${ }^{1}$

\section{Repeated measurements}

The Tinnitus Tester was repeated for 31 subjects after an interval of 2-4 weeks. For 14 of these subjects, the second tinnitus measurement was taken 1-2 weeks after their RI function had been determined. In addition, these subjects played their optimal masking sound (determined by the RI function) several times daily between the Tinnitus Tester sessions using a CD prepared for this purpose. Neither procedure was performed between the repeated Tinnitus Tester sessions for the remaining 17 subjects. For these subjects, the first RI function was measured approximately 1 week after a second session with the Tinnitus Tester had been completed. These data provided information on the test-retest reliability of the tinnitus spectrum and whether it was altered by listening to masking sounds.

The RI function was measured a second time after an interval of 2 weeks in 34 subjects. All of these subjects listened to masking sounds prepared for them between the two RI measurements.

\footnotetext{
The Familiarization Program, Tinnitus Tester, and RI Tester can be downloaded for research use at http://www.psychology. mcmaster.ca/hnplab.
}

\section{Data analysis}

Mean audiograms, tinnitus spectra (the likeness ratings from step 7 of the Tinnitus Tester), and RI functions (RI depth and duration, from step 3 of the RI Tester) were determined for groups of subjects reporting tonal, ringing, or hissing tinnitus. RI functions reported by subjects with bilateral tinnitus were similar for the two ears and were therefore collapsed over ears. Statistical analyses were conducted using Statistica (version 6.0). Nonparametric Friedman analyses of variance by ranks $\left(\chi^{2}\right)$ were used to assess one-way effects of frequency on rated variables (the tinnitus spectrum and RI depth). Analysis of variance with Greenhouse-Geisser correction for repeated measures (general linear model of Statistica) was used for all other analyses including those requiring group comparisons. Significant main effects and interactions were described by least significant difference tests. Significance level was set at $\alpha=0.05$ (two-tailed)

\section{RESULTS}

Using sound clips in the Tinnitus Tester as examples, $53.3 \%(48 / 90)$ of the subjects described their tinnitus as tonal, $25.5 \%(23 / 90)$ as ringing, and $21.1 \%(19 / 90)$ as hissing. Seventy-eight subjects $(86.6 \%)$ reported a continuous stable tinnitus sensation, and the remaining 12 subjects $(13.3 \%)$ a steady pulsating tinnitus sensation. Fifty-nine subjects $(65.5 \%)$ reported bilateral tinnitus and 31 subjects $(34.4 \%)$ unilateral tinnitus (17 right ear). All forms of tinnitus (tonal, ringing, and hissing) were represented within the bilateral and unilateral groups. We present results from the 59 subjects reporting bilateral tinnitus first and then consider the unilateral cases.

\section{Bilateral tinnitus}

Tinnitus spectra, RI functions, and audiograms did not differ significantly between bilateral subjects reporting continuous $(47 / 59)$ and steady pulsing $(12 / 59)$ tinnitus. The two types of subjects were therefore combined for the analyses reported below.

The mean audiogram, tinnitus spectrum (likeness ratings), and functions for RI depth collapsed over tinnitus bandwidth are presented in Figure 2A separately for the cohorts tested in Hamilton and in Vancouver, and for the cohorts combined in Figure 2B. The "audiometric edge" of normal hearing (the stimulus frequency adjoining the frequency at which the hearing threshold exceeded $25 \mathrm{~dB}$ HL) is demarcated by an arrow in each panel. Likeness ratings increased with the depth of hearing loss in each cohort 
(Hamilton: $\chi^{2}(47,10)=123.3, p<0.0000$; Vancouver: $\chi^{2}$ $(12,10)=32.4, p<0.0005)$, exceeding a rating of 40 near $3 \mathrm{kHz}$ in both samples. This rating on the Borg CR100 scale corresponded to a sensation beginning to resemble the tinnitus. Likeness ratings reached asymptote at $6 \mathrm{kHz}$ and persisted into the region of hearing loss in the group-averaged data, with no apparent diminution except at the highest frequency tested $(12 \mathrm{kHz}$, which several subjects could not hear). RI depth followed a similar course in each cohort, increasing at and above $2 \mathrm{kHz}$ with the degree of hearing impairment, reaching asymptote near $6 \mathrm{kHz}$ and persisting above this frequency. Ratings of RI depth differed among the frequencies in both cohorts (Hamilton: $\chi^{2}(47,10)=46.8, p<0.0000$; Vancouver: $\left.\chi^{2}(12,10)=20.3, p<0.026\right)$. RI depth tended to be greater in the Vancouver sample where sound intensities tended to be higher but comparison of the two cohorts by analyses of variance found no differences between them in RI depth or in the tinnitus spectra. Figure 2 also shows RI depth induced by the $\mathrm{WN}$ masker in each panel. RI induced by WN was greater than RI induced by BPN15 maskers with CFs of 0.5 or $1.0 \mathrm{kHz}(p<0.016)$ but less than that induced by BPN15 maskers with CFs between $5-10 \mathrm{kHz}(p<$ 0.028 , LSD tests, cohorts combined). These results indicate that sounds that contained frequencies covering and restricted to the tinnitus spectrum produced greater RI than sounds that did not. Because no differences were found between the Hamilton and Vancouver cohorts in these measures, the two cohorts were combined herein except where otherwise indicated.

Tinnitus spectra are compared across tinnitus bandwidths (tonal, ringing, and hissing) in Figure 3A. Tonal $(n=34)$ and ringing $(n=13)$ tinnitus evoked higher mean likeness judgments than did hissing $(n=$ 12) tinnitus at frequencies between 5 and $10 \mathrm{kHz}$. This effect was significant when the maximum likeness ratings given by each subject regardless of center frequency ("peak" likeness, each peak determined by an average of three trials) were contrasted across the tinnitus bandwidths. Peak likeness ratings obtained for tonal and ringing tinnitus were higher than for hissing tinnitus (mean ratings of 76.6, 74.8, and 60.8, respectively; $F(2,56)=8.06, p=0.006]$ and corresponded to sound frequencies that were higher for tonal (mean, $7.7 \mathrm{kHz}$ ) and ringing (mean, $7.3 \mathrm{kHz}$ )
A
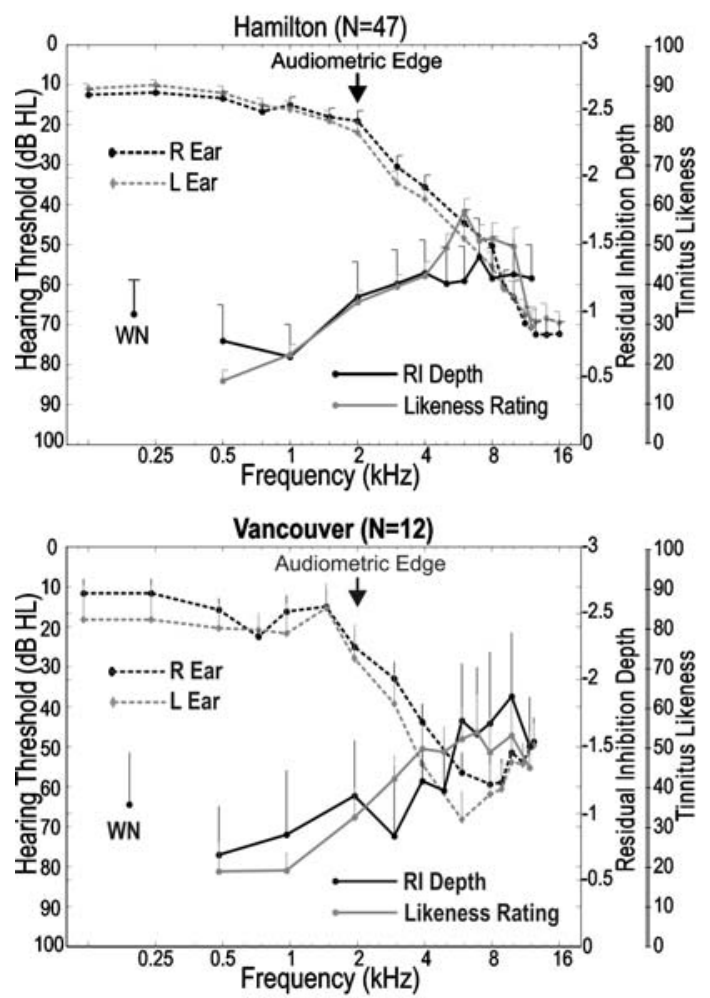

FIG. 2. RI functions and tinnitus spectra inversely track the region of threshold shift in bilateral tinnitus. A Hamilton and Vancouver cohorts are shown separately. B Grand averaged results. The data point to the left in each panel gives RI depth induced by white noise $(W N)$. The

B

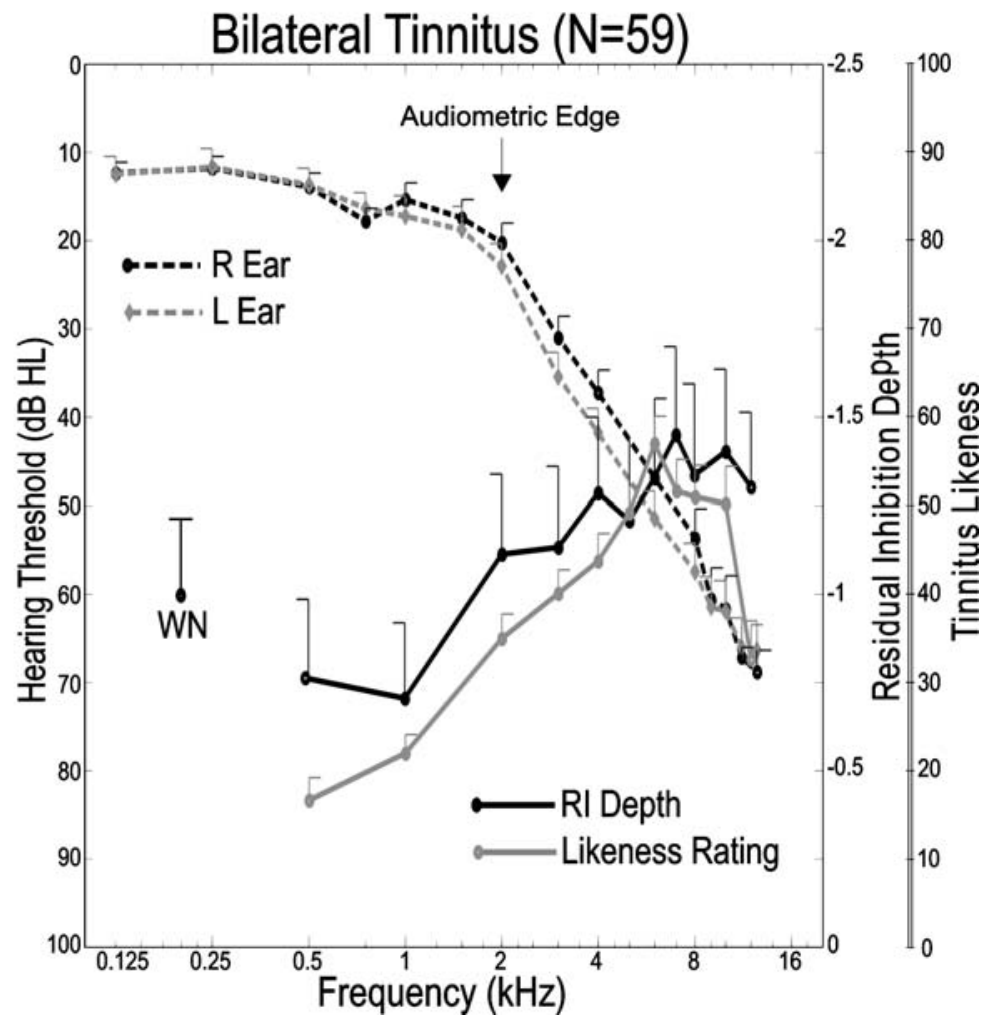

arrow denotes the "audiometric edge" of normal hearing (the stimulus frequency adjoining the frequency at which the hearing threshold falls above $25 \mathrm{~dB} \mathrm{HL}$ on the audiometrically reversed $\mathrm{dB}$ axis). Error bars denote 1 standard error in this and subsequent figures. 
A

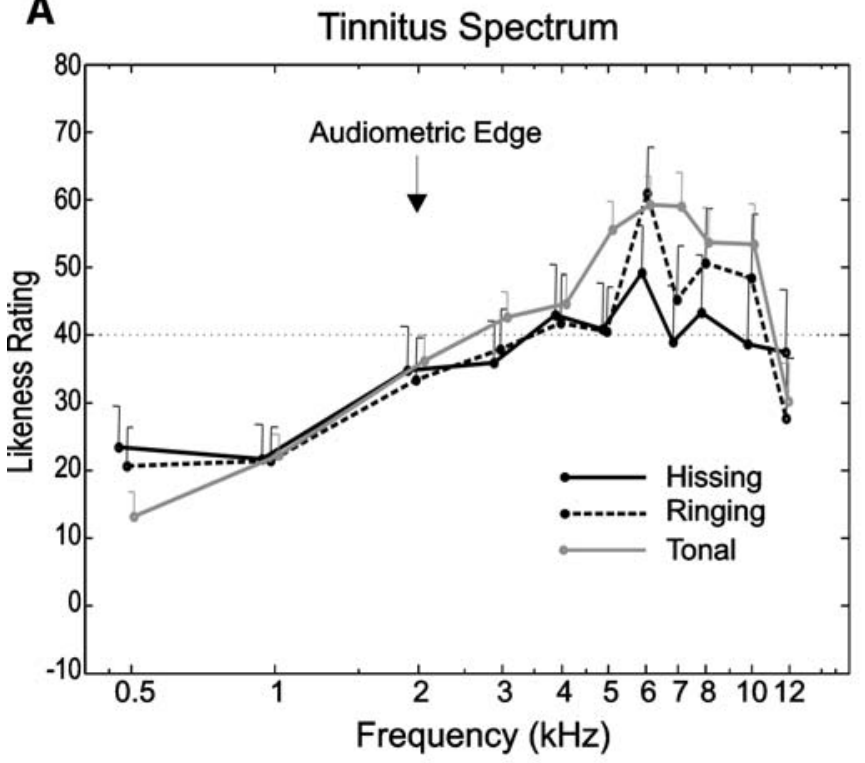

C

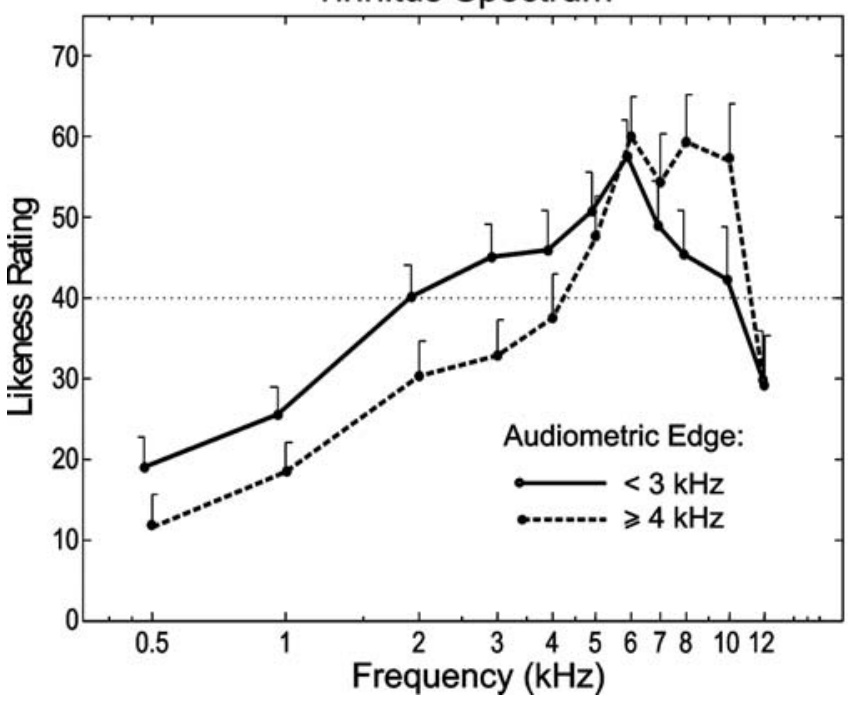

FIG. 3. Tinnitus spectra and loudness. A Tinnitus spectra vary with tinnitus bandwidth (tonal, ringing, and hissing tinnitus). The broken line denotes a rating of 40 on a Borg CR100 scale, indicating that the sound is beginning to resemble the tinnitus. B The tinnitus spectrum peaks at $6 \mathrm{kHz}$ in poorer hearing tinnitus subjects but continues to rise to higher frequencies in better hearing tinnitus subjects (tinnitus bandwidths combined). Better hearing subjects had hearing thresholds $\leq 35 \mathrm{~dB} \mathrm{HL}$ at $8 \mathrm{kHz}$ (poorer hearing subjects had hearing thresholds $>35 \mathrm{~dB} \mathrm{HL}$ at $8 \mathrm{kHz}$ ). C The tinnitus spectrum covered

tinnitus compared to hissing tinnitus (mean, $5.4 \mathrm{kHz}$; $p=0.034$ ). The tinnitus spectrum was also influenced by hearing status. Two analyses were conducted, collapsing over tinnitus bandwidth in each. In one analysis (Fig. 3B), spectra were contrasted when bilateral cases were classified into two subgroups, one subgroup with comparatively good hearing up to $8 \mathrm{kHz}$ (hearing thresholds $\leq 35 \mathrm{~dB} \mathrm{HL}$ at this frequency, $n=11$ ) and another with comparatively poorer hearing (thresholds $>35 \mathrm{~dB}$ at $8 \mathrm{kHz}, n=48$ ).
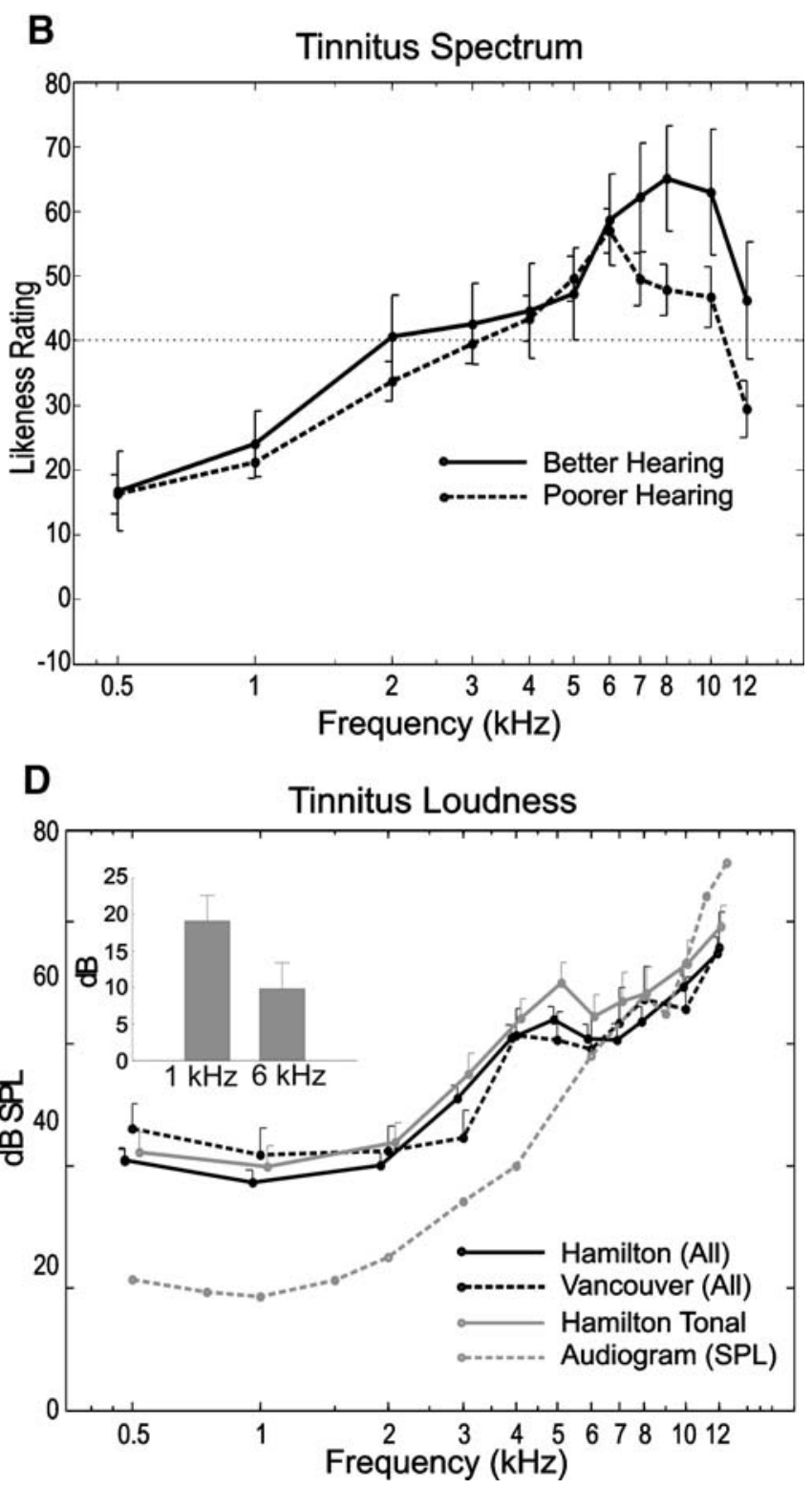

higher frequencies when the audiometric edge was above $4 \mathrm{kHz}$. The three tinnitus bandwidths are combined. D Tinnitus loudness is greater when matched using sound frequencies outside of the region of hearing loss. Tinnitus loudness matches are shown separately for the Hamilton and Vancouver cohorts (averaged over tinnitus bandwidth). Loudness matches for tonal cases and their audiogram converted to SPL are also given for the Hamilton sample only. The inset contrasts the difference between the latter two functions at 1 and $6 \mathrm{kHz}$ (tinnitus loudness).

The better hearing subgroup gave likeness ratings that increased incrementally up to $10 \mathrm{kHz}$, whereas the poorer hearing group gave likeness ratings that peaked at $6 \mathrm{kHz}$ and declined at higher frequencies (see Fig. 3B; group contrast $F(1,56)=4.00$ for the frequency range $7-12 \mathrm{kHz}, p=0.05)$. All but one of the subjects in the better hearing group $(10 / 11)$ were narrow band (tonal or ringing) tinnitus cases. In the second analysis, subjects were divided into subgroups according to the edge of normal hearing in the 
audiogram. The tinnitus spectrum covered higher frequencies in subjects for whom the audiometric edge was at or above $4 \mathrm{kHz}(n=28)$ compared to subjects with audiometric edges below this frequency ( $n=31$, interaction of frequency with subgroup $F(9$, $396)=2.60, p=0.006$, see Fig. 3C) .

Figure 3D shows the loudness matches in $\mathrm{dB}$ SPL obtained from step 6 of the Tinnitus Tester. In this procedure, subjects adjusted the loudness of sounds of different CFs to equal the loudness of their tinnitus, using sounds chosen by the subject to approximate the bandwidth of their tinnitus. Loudness matches shown for the Hamilton and Vancouver cohorts overlapped extensively and did not differ at any frequency. Although no main effect of tinnitus bandwidth was found, loudness matches were on average $15 \mathrm{~dB}$ lower for hissing tinnitus than for tonal and ringing tinnitus above $5 \mathrm{kHz}(p=0.048$, data not shown), suggesting that loudness matches given by subjects with hissing tinnitus were based to some degree on listening to the low-frequency content of the BPN15 maskers these subjects received. Also shown separately in Figure 3D are the loudness matches obtained for subjects reporting tonal tinnitus in the Hamilton sample and the average audiogram for these subjects converted to SPL using calibration curves for pure tones at threshold delivered by HDA 200 headphones (Han and Poulsen 1998). The difference between these functions gives an estimate of the loudness of tinnitus (in dB SL) obtained by matching techniques. Tinnitus loudness was greater when matched using frequencies below the tinnitus spectrum (19.1 dB at $1 \mathrm{kHz})$ than when using frequencies in the spectrum $[9.8 \mathrm{~dB}$ at $6 \mathrm{kHz}, t(29)=$ 3.04, $p=0.005$; see inset Fig. 3D]. This calculation could not be performed for subjects with ringing or hissing tinnitus because hearing thresholds were not available for the BPN5 and BPN15 sounds these subjects heard. These findings corroborate those obtained with conventional techniques, which show that estimates of tinnitus loudness obtained by matching to pure tones are higher for pure tones outside compared to inside the tinnitus spectrum (Henry and Meikle 2000).

RI depth and duration are presented separately in Figure 4A for the three different tinnitus bandwidths (Hamilton and Vancouver cohorts combined). Because all subjects received BPN15 maskers during the measurement of RI functions regardless of their type of tinnitus, the matching of tinnitus and masker bandwidth could reasonably be assumed to be better for hissing cases than for tonal or ringing tinnitus, which might favor enhanced RI. The results for RI depth were consistent with this view (Fig. 4A, left panel). A main effect of $\mathrm{CF}$ was found $[F(10,550)=$ $4.10, p=0.004]$, which was attributable to greater RI depth for CFs between 3-10 kHz than for CFs below this range $(p=0.005)$. Although the main effect of tinnitus bandwidth did not reach significance, a contrast comparing RI depth for subjects with hissing tinnitus with the ringing and tonal groups gave $F$ $(1,55)=4.99, p=0.029$ at $6 \mathrm{kHz}$, and approached significance for the frequency range $4-10 \mathrm{kHz}(\mathrm{p}=$ 0.10 ). The peak level of RI depth regardless of CF also tended to be greater for subjects with hissing tinnitus compared to the other types $(\mathrm{p}=0.08)$. RI duration (Fig. 4A, right panel) also showed a main effect of CF $[F(10,550)=4.31, p=0.003]$, which reflected longer lasting RI for CFs in the range of $3-10 \mathrm{kHz}(p=0.005)$ than for lower CFs. However, no effect of tinnitus bandwidth on RI duration was found.

Figure 4A indicates that overall mean RI depth was shallow (mean, $2-12 \mathrm{kHz}=-1.28$ on a five-point scale, $p=0.0000$ ) and RI duration brief (mean, 2$12 \mathrm{kHz}=16.7 \mathrm{~s}, p=0.0000)$. There was, however, large variability between subjects in these measures. To depict this variability, we classified our 59 bilateral cases into six categories corresponding to peak RI depth and into five categories according to peak RI duration (both peak measures the average of three trials, representing the maximum at any $\mathrm{CF}$ ). Figure 4B shows that $13 / 59$ subjects $(22.0 \%)$ reported peak RI depth at or approaching tinnitus elimination (scale ratings of -4.0 to $-5.0,-5.0$ meaning "tinnitus gone"), and $11 / 59(18.6 \%)$ reported peak RI duration between 35 and $45 \mathrm{~s}$ (the time-out limit was $45 \mathrm{~s}$ ). Three subjects reported tinnitus elimination or nearelimination after all maskers, suggesting that suppression induced by the maskers persisted at least throughout RI testing (about $1 \mathrm{~h}$ ). Follow-up reports volunteered by three subjects given maskers for home use described RI lasting longer (up to $20 \mathrm{~min}$ ) after repeated masker use. In contrast, 5/59 subjects $(8.5 \%)$ reported small increases in tinnitus after the masking stimuli, and 3/59 subjects $(5.1 \%)$ peak RI durations of less than $5 \mathrm{~s}$. The majority of subjects fell between these extremes. Overall, 41/59 subjects $(69.5 \%)$ reported peak RI depths exceeding -1.0 ( $20 \%$ of scale) following one or more masking stimuli. The CF of these maskers was typically above $2 \mathrm{kHz}$ (see Fig. 2).

We subsequently partitioned our 59 bilateral subjects into two subgroups on the basis of RI depth in order to identify predictors of RI. All subjects who gave depth ratings $\leq-2.0$ for at least one masker $\mathrm{CF} \geq$ $4 \mathrm{kHz}(n=30)$ were classified as "good RI," and the remaining subjects $(n=29)$ as "poor RI." The two subgroups are contrasted in Figure 5A where main effects for CF were found for RI depth $(p=0.000)$ and duration $(p<0.001)$ within the good-RI subgroup, reflecting deeper and longer lasting RI for BPN15 maskers with high CF in this subgroup. However, little 
A

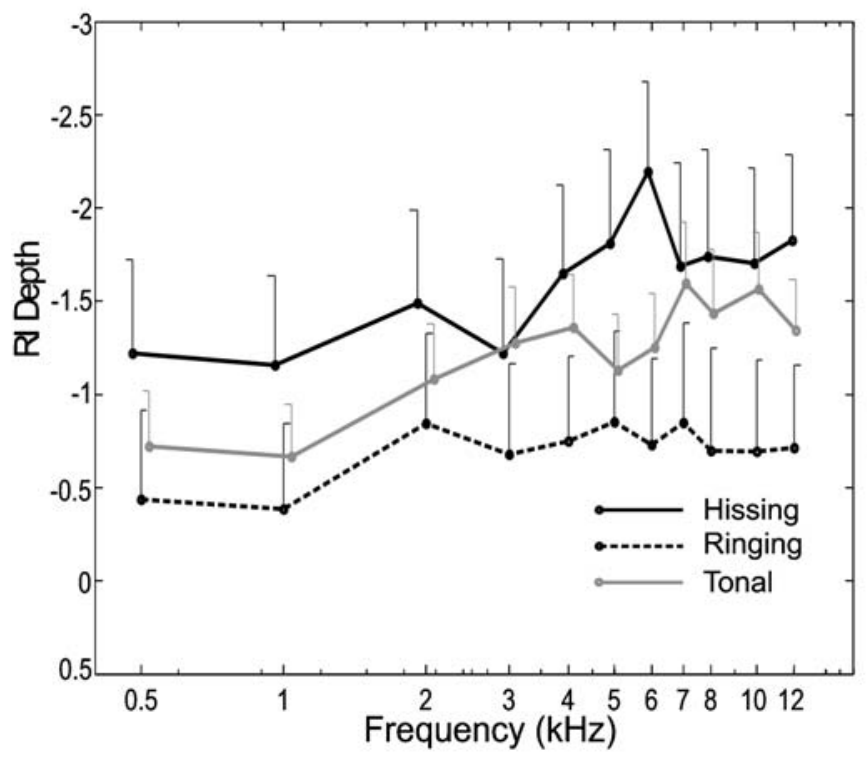

B

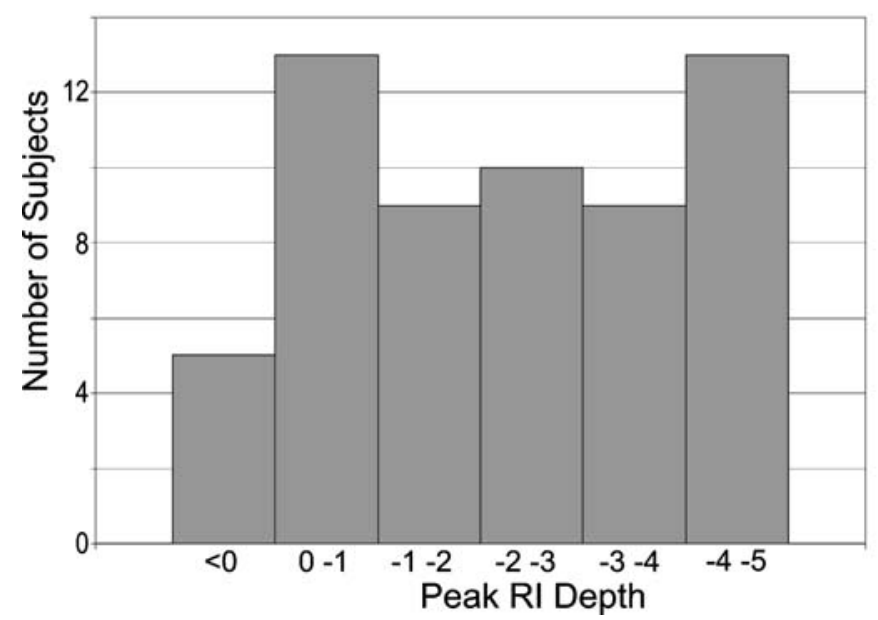

FIG. 4. RI functions for depth and duration reach their maxima in and cover the region of hearing loss $(2-10 \mathrm{kHz}$ ). A Mean RI depth (left panel) and RI duration (right panel) for the three tinnitus bandwidths.

RI was evident in the poor-RI subgroup. Figure 5B contrasts the two subgroups for RI masker loudness in dB SPL (from step 2 of the RI tester, where subjects adjusted the loudness of each BPN15 sound to equal a $1-\mathrm{kHz} 65 \mathrm{~dB}$ SL pure tone up to a limit of $\sim 95 \mathrm{~dB}$ SPL). Tinnitus loudness in SPL is also shown in the figure (from step 6 of the Tinnitus Tester, where each subject adjusted a sound to equal the loudness of their tinnitus). These results show that RI masker loudness was on average $10.0 \mathrm{~dB}$ louder in the good RI subgroup at all CFs compared the poor RI subgroup (main effect of subgroup $F(1,57)=6.73, p=$ 0.012 ). In addition, tinnitus was on average $7.2 \mathrm{~dB}$ quieter in the good-RI subgroup at frequencies below $4 \mathrm{kHz}$ compared to poor-RI subjects (subgroup $\times \mathrm{CF}$
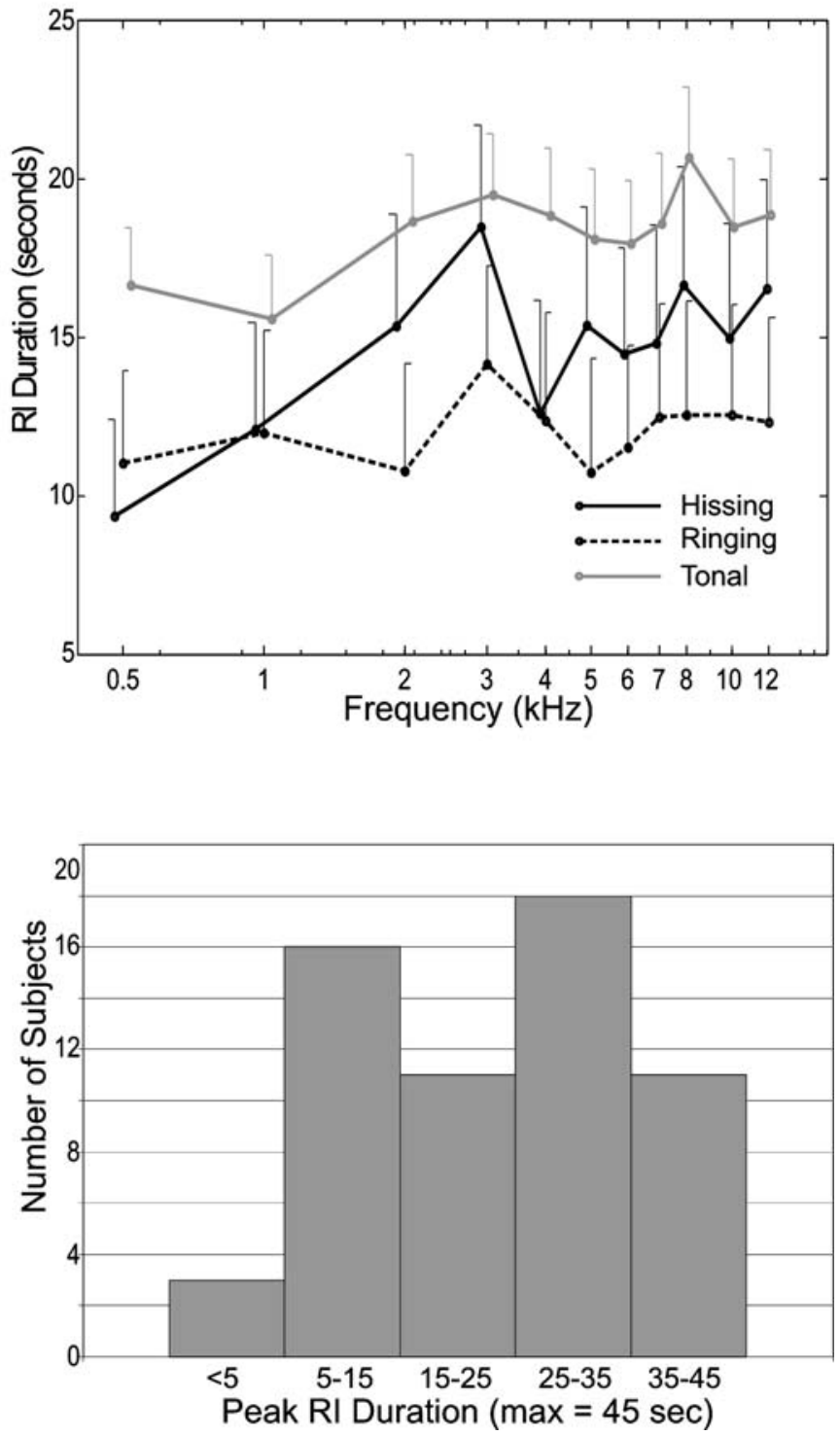

Sample size is $n=12,13$, and 34 subjects in the hissing, ringing, and tonal subgroups, respectively. B Subjects are distributed according to peak RI depth and peak RI duration ( $n=59$ bilateral cases).

interaction $[F(10,570)=2.31, p=0.011]$. These effects combined to determine the variable "masking level" (the difference between tinnitus loudness and RI masker loudness in $\mathrm{dB}$ ), which is shown separately for each group in Figure 5C. Masking level was on average $12.7 \mathrm{~dB}$ higher in the good-RI group $[F(1,57)$ $=8.28, p=0.006]$ and decreased with $\mathrm{CF}$ in both groups $[F(10,570)=48.4, p=0.000]$, with a non-significant subgroup by $\mathrm{CF}$ interaction. At CFs above $4 \mathrm{kHz}$, masking level averaged $13.3 \mathrm{~dB}$ in the good-RI subjects compared to $2.9 \mathrm{~dB}$ in the poor-RI subjects ( $p=$ $0.035)$. These results suggest that RI was impaired in poor-RI subjects because the BPN15 sounds they received above $4 \mathrm{kHz}$ did not adequately mask their tinnitus. Hearing thresholds did not differ between 
A

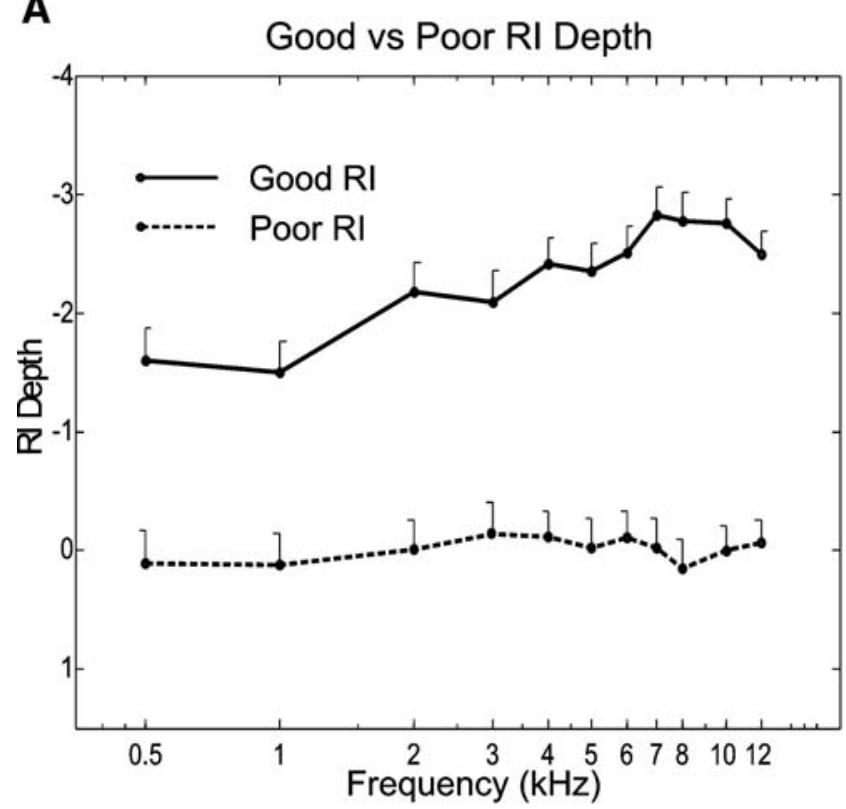

B

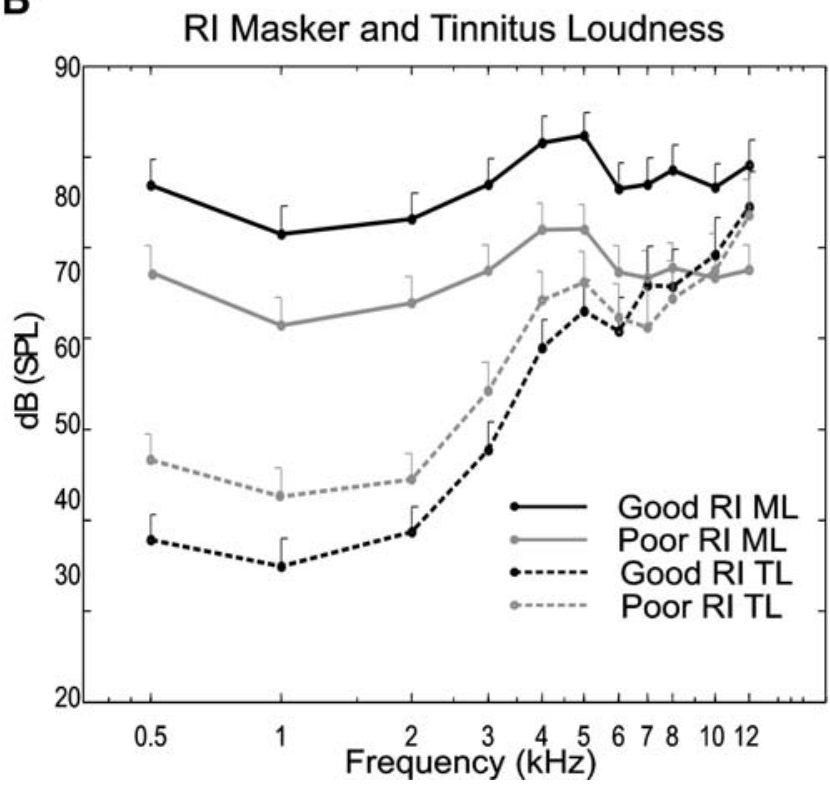

FIG. 5. RI depth increases with masking level. A Contrast of subgroups with good RI (RI depth $\leq-2.0)$ and poor RI, for RI depth and RI duration. The poor RI group does not show significant RI depth (mean rating is zero across CFs). B RI masker loudness $(M L)$

the good-RI and poor-RI groups, although a slightly lower threshold $(5.5 \mathrm{~dB})$ for the $1 \mathrm{kHz}$ reference tone in poor-RI subjects $(p=0.15)$ may have contributed to inadequate masking in this group. A further finding was that the good-RI group gave higher tinnitus likeness ratings for sounds above $4 \mathrm{kHz}$ than did the poor-RI group $[F(1,57)=5.19, p=0.026$, results not shown], which suggests that the sounds presented by the Tinnitus Tester were perceived to more closely approximate the tinnitus sensation in good-RI compared to poor-RI subjects.

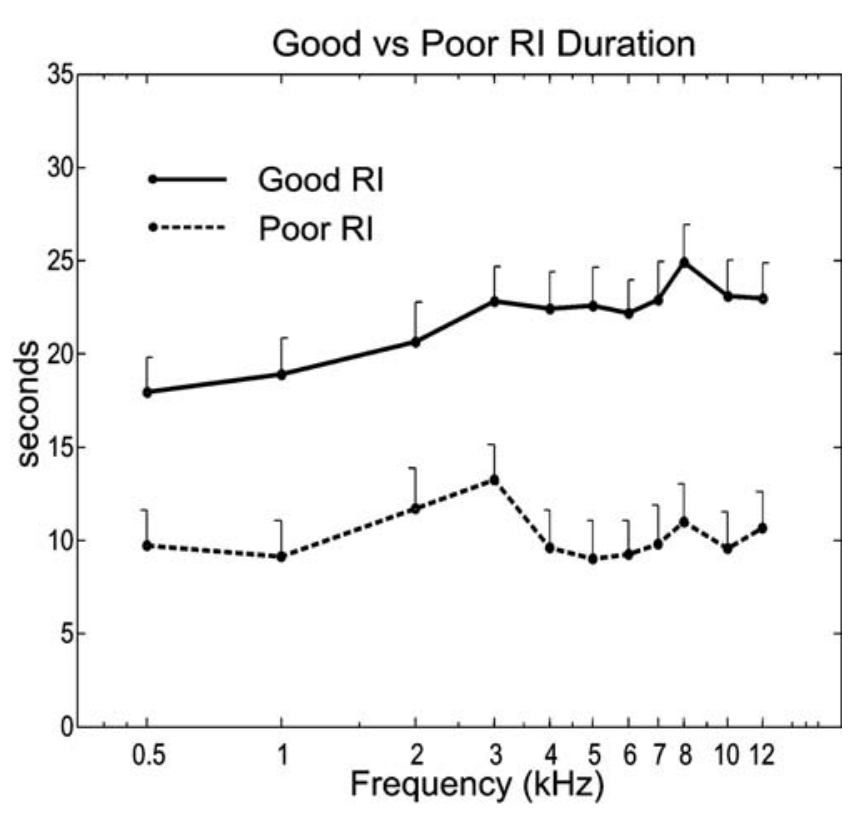

C

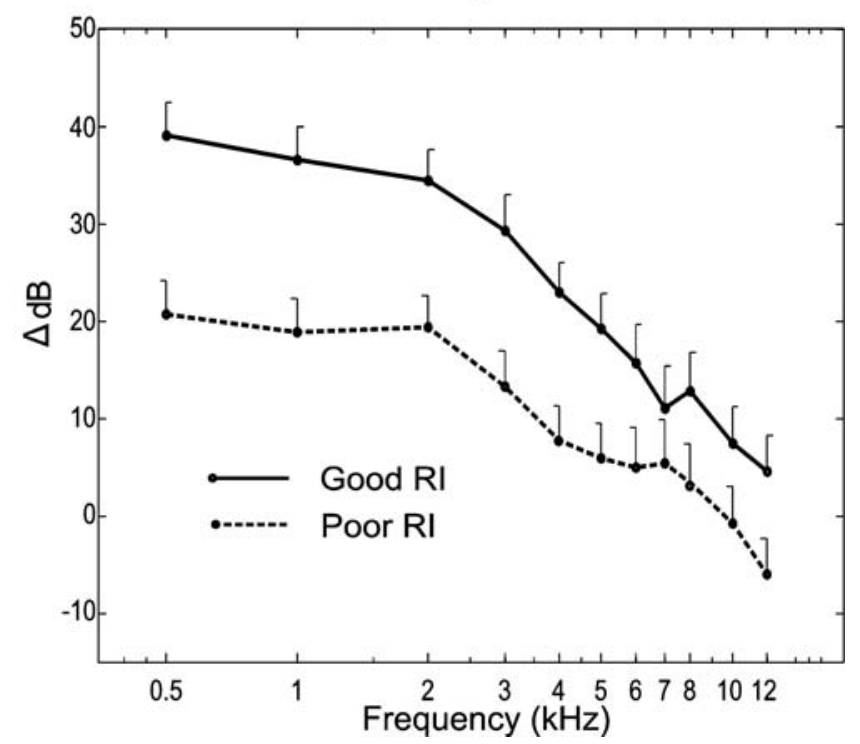

and tinnitus loudness $(T L)$ in SPL, in the good RI and poor RI subgroups. C Mean masking level is higher in the good compared to the poor RI subgroup. Masking level is the difference between RI masker loudness and tinnitus loudness in $\mathbf{B}$.

\section{Unilateral tinnitus}

Thirty-one of the 90 subjects $(34.4 \%)$ in the combined cohort reported tinnitus perceived only in one ear. Tinnitus spectra and RI functions determined for the affected ear are presented in Figure 6 separately for left $(n=14)$ and right $(n=17)$ ear tinnitus including the mean audiogram obtained for each tinnitus type. Tinnitus spectra and RI functions for depth and duration (only RI depth is shown in Fig. 6) did not differ between left and right ear tinnitus and 

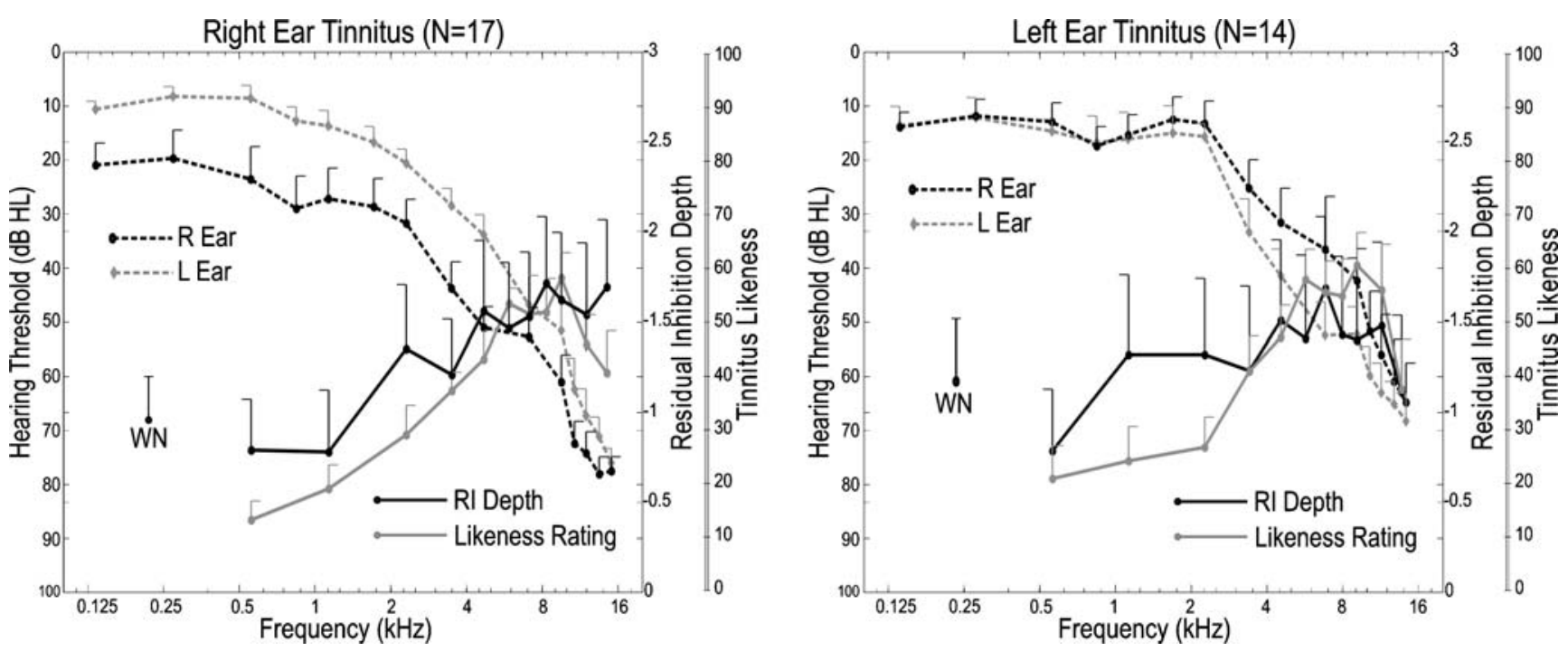

FIG. 6. RI functions and tinnitus spectra inversely track the region of threshold shift in unilateral tinnitus. The format is the same as in Figure 2 .

were similar to the spectra and RI functions found in bilateral tinnitus, which reached their asymptotes at frequencies covering the range of hearing loss. Of the 31 unilateral cases, six subjects $(19.4 \%)$ reported peak RI depth approaching tinnitus elimination ( -4.0 to -5.0$)$ and eight subjects $(25.8 \%)$ peak RI duration between $35-45$ s, similar to percentages observed for bilateral tinnitus (22.0\% and $18.6 \%$, respectively). One noteworthy subject reporting tinnitus in the left ear displayed hearing thresholds of $40 \mathrm{~dB}$ HL in both ears between 4$6 \mathrm{kHz}$ but normal thresholds ( $<25 \mathrm{~dB}$ HL) above and below this range (a notched audiogram, suggesting hearing loss induced by noise exposure). Although in the range of normal hearing, thresholds outside of the notch were $\sim 10 \mathrm{~dB}$ higher in the left (tinnitus) ear compared to the right ear above $8 \mathrm{kHz}$. The tinnitus spectrum and RI function for this subject reached asymptote at $4-6 \mathrm{KHz}$ and diminished above and below this frequency range, corresponding to the region of the notch.

Although subjects reporting left or right unilateral tinnitus did not differ with respect to tinnitus spectra or RI functions in the affected ear, in 22 of 31 subjects, hearing thresholds were elevated by $10 \mathrm{~dB}$ or more in the ear ipsilateral to the tinnitus [Fig. 6, interaction of tinnitus type and ear; $F(1,29)=15.9, p<$ $0.0005)$. For left-ear tinnitus, hearing thresholds were elevated by $10.9 \mathrm{~dB}$ between $3-10 \mathrm{kHz}$ in the left compared to the right ear $(p=0.004)$, whereas in rightear tinnitus, hearing thresholds at these frequencies as well as at lower frequencies were similarly elevated in the right relative to the left ear $(p=0.003)$. Neardeafness in the right ear of three subjects was responsible for the wider frequency range of impairment in right ear tinnitus. On the other hand, in bilateral subjects (Fig. 2), hearing function did not differ between the ears. Hence, the relative degree of hearing loss between the ears corresponded to whether subjects experienced unilateral left, unilateral right, or bilateral tinnitus.

\section{Role of hearing loss and age}

The relation of relative hearing loss between the ears to unilateral and bilateral tinnitus implicates hearing impairment as a factor in the generation of tinnitus and in its suppression during RI. To investigate the role of hearing loss further, we measured audiograms for 31 control subjects agematched to the Hamilton bilateral tinnitus group but reporting no tinnitus. Because age has large effect on hearing, age matching was done separately for subjects $\leq 50$ years old (tinnitus $n=7$, mean $34.0 \pm$ 7.3 years; controls $n=9$, mean $34.4 \pm 8.3$ years) and subjects over age 50 years (tinnitus $n=40$, mean $62.1 \pm$ 6.9 years; controls $n=22$, mean $65.6 \pm 4.9$ years). Audiograms for tinnitus and control subjects are compared separately for the two age groups in Figure 7. In the older group, the main effect of tinnitus (tinnitus/ control) on hearing thresholds $[F(1,60)=8.58, p=$ $0.005]$ and the interaction of tinnitus with frequency $[F(16960)=2.38, p=0.0017]$ were significant. Hearing thresholds were higher in tinnitus subjects than in controls mainly at frequencies between $2-8 \mathrm{kHz}(54.8$ vs. $41.9 \mathrm{~dB}$ in tinnitus subjects and controls, respectively; difference $=12.9 \mathrm{~dB}$ ). The audiograms obtained for younger tinnitus subjects were more similar to those obtained for their age-matched controls. While the main effect of tinnitus over all frequencies was not significant, post hoc contrasts found that hearing thresholds were on average $11.1 \mathrm{~dB}$ higher in young 


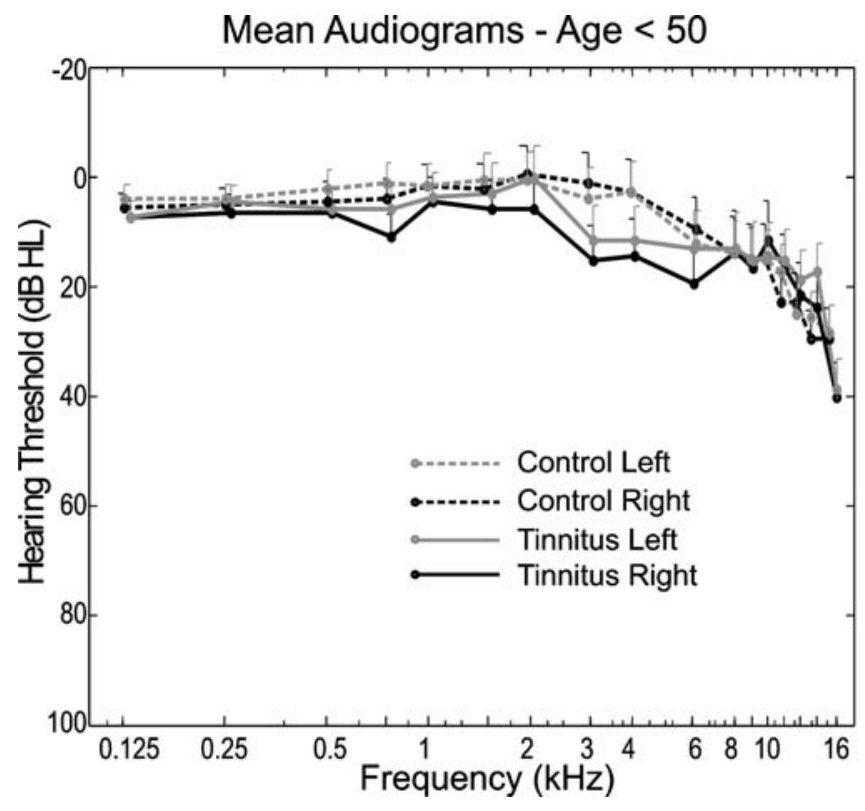

FIG. 7. Hearing thresholds are elevated between $2-8 \mathrm{kHz}$ in tinnitus subjects compared to age-matched controls. This is true for younger subjects (age $\leq 50$ years) as well as older subjects (age $>50$ years),

tinnitus subjects $(13.4 \mathrm{~dB})$ than in the controls ( $2.3 \mathrm{~dB})$ between $2-4 \mathrm{kHz}(p \leq 0.044)$, although mean thresholds were still within in the range considered normal ( $\leq 25 \mathrm{~dB} H \mathrm{HL})$ in clinical audiology.

Because their hearing function was different, we contrasted the two age groups of tinnitus subjects for RI depth, tinnitus spectra, and tinnitus bandwidth. Tinnitus spectra resembled those in Figure 2 and did not differ between the two age groups. The majority of subjects in each subgroup reported tonal or ringing tinnitus with no notable group difference on this variable. However, a difference in RI depth was found $[F(1,45)=5.27, p=0.026]$, in which older tinnitus subjects showed typical RI functions in which RI depth increased with $\mathrm{CF}$, whereas younger subjects reported little change or small increases in tinnitus after exposure to masking stimuli with CFs between 4$10 \mathrm{kHz}$. The RI functions for subjects aged $\leq 50$ years closely resembled those of poor RI subjects described in Figure 5A and did not differ from them at any CF. However, for subjects aged $\leq 50$ years, poor RI could not be attributed to low levels of masking. On the contrary, the masking levels received by these subjects during RI testing averaged near $20 \mathrm{~dB}$ at all CFs and were higher than those of older subjects between 3$10 \mathrm{kHz}$ where RI was most likely to be experienced (20.3 vs. $7.2 \mathrm{~dB}$ in the two age groups, respectively, in this frequency range, $p=0.04$ ). Masking levels were higher in younger than older subjects, in part because, for older subjects, RI masker loudness often reached the allowable limit of $95 \mathrm{~dB}$ SPL at higher

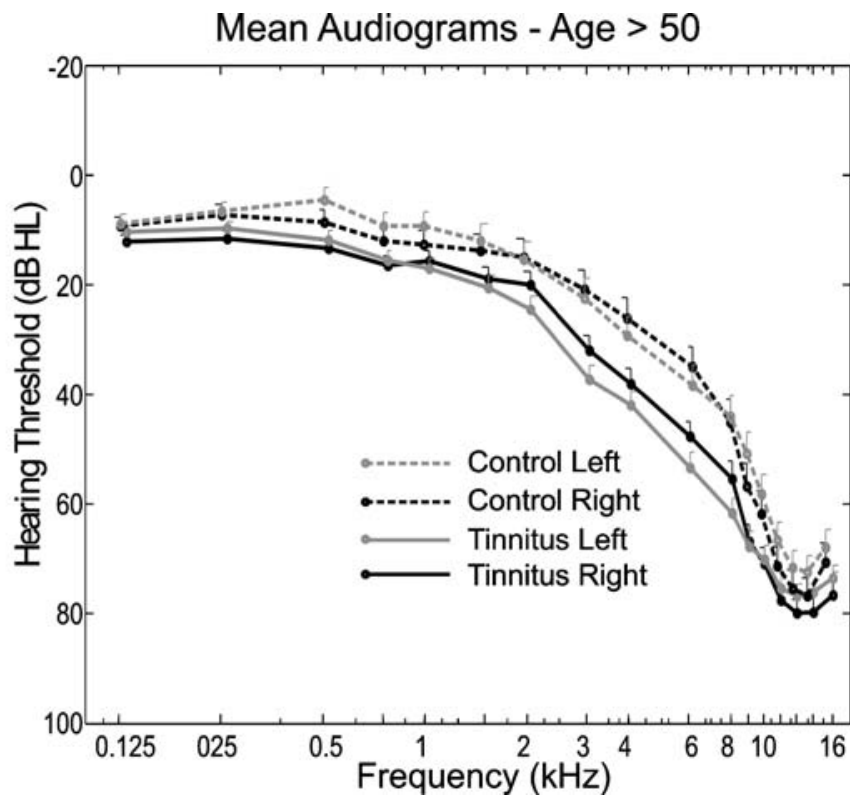

even though the mean audiogram for younger subjects is in the normal range up to $10 \mathrm{kHz}$.

frequencies. In addition, the tinnitus loudness matches of younger subjects were lower than those of older subjects above $3 \mathrm{kHz}$ [group $\times$ frequency interaction $F(10,450)=11.1, p=0.0000$, results not shown].

These results suggest that age as well as masking level may have influenced RI depth. Multiple regression applied to RI depth averaged over $4-10 \mathrm{kHz}$ for all tinnitus subjects $(n=90)$ uncovered a significant contribution from both factors $(\beta=-0.25$ and -0.34 for masking level and age, respectively, $p<0.05)$. Hearing thresholds (averaged $4-10 \mathrm{kHz}$ for the tinnitus ears) also contributed significantly ( $\beta=-0.29$, $p=0.05$ ) to RI depth but only if the age variable was removed, reflecting a confounding of these factors. When RI depth was related to masking level for all bilateral cases collapsing across $4-10 \mathrm{kHz}$, a secondorder polynomial gave the best although poor fit to the data ( $4 \%$ of the variance accounted for), revealing a shallow curvilinear relationship owing to little RI reported by younger subjects where masking level was comparatively high. When the younger subjects were removed, RI depth increased linearly with masking level $(r=-0.303, p<0.05)$.

\section{Repeated measurements}

Thirty-one subjects distributed across the tinnitus types (ear and bandwidth) returned for a second measurement with the Tinnitus Tester after an interval of 2-3 weeks. Of these subjects, 14 had their 
RI functions determined and were provided with maskers for home listening between tinnitus measurements. Main effects of home use and test-retest were not significant for tinnitus spectra or tinnitus loudness. Tinnitus spectra and loudness matches obtained at the second measurement closely overlapped those of the first measurement across frequencies and did not differ significantly between measurements at any frequency. Between-subject correlations calculated for each stimulus frequency ranged from 0.12 to 0.80 (mean $r=0.39, p<0.0003$ ) for the likeness judgments and from 0.33 to 0.78 (mean $r=0.64, p<0.000$ ) for the loudness matches.

Test-retest information on tinnitus bandwidth was available for 27 subjects. Seventeen subjects (63\%) gave the same tinnitus bandwidth classification on the second test. Hissing tinnitus was the most reliable type ( $n=5,80 \%$ unchanged) followed by tonal tinnitus ( $n=$ $15,67 \%$ unchanged) and ringing tinnitus ( $n=7,43 \%$ unchanged). Eighty percent of changes between sessions were to the adjacent bandwidth. There were no shifts in tinnitus ear between sessions.

Thirty-four subjects with bilateral tinnitus returned for a second determination of their RI functions after an interval of 2-3 weeks. During this interval, all subjects played their optimal masking sound several times daily, using a CD prepared for this purpose. RI functions for depth and for duration at retest were highly similar between sessions, increasing with the depth of hearing loss and reaching asymptote for masking sounds covering the range $4-10 \mathrm{kHz}$ (main effects of test-retest on depth and duration were not significant). Between-subject correlations calculated for each stimulus ranged from 0.51 to 0.77 (mean $r=$ $0.60, p<0.000)$ for RI depth and from 0.30 to 0.52 (mean $r=0.41, p<0.000$ ) for RI duration.

It will be recalled that a brief test for RI was administered at the end of the Tinnitus Tester. In this test, RI induced by the $5.0 \mathrm{kHz}$ BPN15 masker $(p=0.0008)$ and the $0.5 \mathrm{kHz}$ BPN15 masker $(p=0.001)$ was superior $(p=0.009)$ to that induced by the custom masker, which did not give significant RI $(p=0.45)$ when the three tinnitus bandwidths were combined. However, a contrast comparing RI depth induced by the custom masker between hissing tinnitus (mean $=-1.32$ ) and the other two tinnitus types (mean $=0.60$ and -0.22 for ringing and tonal, respectively) was significant $[F(2,42)=3.98, p=0.05]$, indicating that the single pure tones or BPN5 maskers that were used respectively for tonal and ringing tinnitus did not give significant RI but that the custom masker did so for subjects with hissing tinnitus. The correlation of RI depth between the Tinnitus Tester and the RI Tester was $r=0.52$ for the $0.5 \mathrm{kHz}$ BPN15 masker and $r=0.36$ for the $5.0 \mathrm{kHz}$ BPN15 masker (both $r$ 's $p<0.05$ ).

\section{DISCUSSION}

Studies of the pathophysiology of tinnitus have suggested that tinnitus is generated when synchronous activity develops among neurons in frequency regions of the primary auditory cortex that have been partially deafferented owing to hearing loss induced by noise exposure or by the aging process (Eggermont and Roberts 2004). This hypothesis implies (i) that the tinnitus percept should consist of a spectrum of sound frequencies that covers the region of impaired hearing in the audiogram, and (ii) that "residual inhibition functions" that relate residual suppression of tinnitus to the properties of masking sounds will reach their maxima for sounds that cover the tinnitus spectrum and the region of hearing impairment. The latter prediction is based on the assumption that such sounds inject excitation and comparatively stronger feed-forward inhibition into the affected cortical regions when presented at suprathreshold levels (Cruikshank et al. 2007), briefly disrupting the synchronous network activity underlying the tinnitus sensation.

Our findings confirmed both of these predictions when the psychoacoustic properties of tinnitus were assessed by automated tools implemented at two independent test sites. Measurement of hearing thresholds in age-matched control subjects without tinnitus further implicated hearing loss as a triggering factor. Elevated hearing thresholds, however, were not a sufficient condition for the experience of tinnitus. In what follows, we first discuss tinnitus spectra and RI functions, and some limitations in their measurement. We then consider the bearing of our results on the mechanism of tinnitus and the role of hearing loss in its generation.

\section{Tinnitus spectra}

The tinnitus spectra reported in Figure $3 \mathrm{~A}$ are congruent with those reported by Noreña et al. (2002) who devised a similar method to assess tinnitus percepts. Noreña et al. asked subjects $(N=10)$ to state whether each of a set of pure tones corresponded to a component of their tinnitus sensation and, if it did, to rate on a ten-point scale the degree to which each sound was part of their tinnitus. For each subject (all reporting tonal tinnitus), tinnitus ratings increased, commencing above the edge of normal hearing, and either spanned the region of hearing impairment to the limit of hearing (eight subjects with presbycusis) or focused on sound frequencies centered in notches in the audiogram (two subjects). In our study, the tinnitus spectrum was measured using sounds that were chosen from a set of three stimuli to most closely resemble the tinnitus bandwidth (tonal, ringing, or 
hissing). In each case, tinnitus likeness ratings increased commencing at the edge of normal hearing in the audiogram (2 kHz, averaged across subjects), and then continued to rise in group-averaged data with the depth of hearing loss (Fig. 2B), reaching a plateau at $6 \mathrm{kHz}$ and persisting to the limit of hearing, which for most of our subjects, was near $10 \mathrm{kHz}$. This spectrum was sensitive to the edge of normal hearing, covering higher frequencies when the audiometric edge equaled or exceeded $4 \mathrm{kHz}$ compared to cases where the limit of normal hearing was equal to or below $2 \mathrm{kHz}$ (Fig. 3C). In agreement with Noreña et al. (2002), subjects with tonal tinnitus judged several pure tones to resemble their tinnitus, such that their mean spectra spanned the region of threshold shift (Fig. 3A). This was not because individual subjects reported single sharp peaks that differed between subjects, which could have blurred the group average. Rather, for the large majority of subjects, several sounds covering the region of hearing impairment were judged to comprise the tinnitus percept.

While these findings are consistent with the neural synchrony hypothesis, a factor to consider is how hearing loss may have affected the perception of sounds that were used to measure the tinnitus spectrum. Such effects are likely to depend on the bandwidth of the sounds that were used. In this respect, it is noteworthy that subjects with hissing tinnitus who heard BPN15 sounds gave peak likeness ratings that were significantly lower than those of subjects with tonal and ringing tinnitus who heard pure tones or narrow band BPN5 sounds. The likeness peaks for hissing subjects also occurred at significantly lower CFs $($ mean $=5.4 \mathrm{kHz})$ than in the other two tinnitus types (mean=7.7 and $7.3 \mathrm{kHz}$ for tonal and ringing tinnitus, respectively). These results suggest that subjects with hissing tinnitus may have listened preferentially to the low-frequency content of the BPN15 maskers they received, where their hearing was better (called "low-frequency listening" herein). If so, these subjects may have experienced a disparity between their perception of the BPN15 sound and their tinnitus, which prevented an improving likeness match to the tinnitus as the CF of the BPN15 sounds increased. On the other hand, low-frequency listening could not have occurred for tonal subjects who received pure tones when the spectrum was measured and was not likely to have occurred for ringing subjects who heard narrow band BPN5 sounds. This interpretation suggests that the tinnitus spectrum of subjects with hissing tinnitus may have more closely resembled that of tonal tinnitus had we used pure tones to measure the spectrum in these cases.

A second factor that may have affected perception of the sounds used for tinnitus measurement is the possible presence of dead regions on the basilar membrane of the cochlea in our subjects. The peaks of psychophysical tuning curves for pure tones presented within a dead region are typically displaced to frequencies adjacent to (or just below) the dead region, indicating that the perception of the pitch of the tones is dependent on the spread of activation on the basilar membrane to adjacent regions where inner hair cell function is better preserved ("off-place listening"; Florentine and Houstsma 1983; Huss and Moore 2005a). Pure tones presented within the range of a dead region are also perceived as distorted or noise-like (Huss and Moore 2005b), which could introduce a further disparity between how subjects hear the sounds presented to measure tinnitus and their tinnitus percept, which may have a more tonal quality. Aazh and Moore (2007) found dead regions to be present in 36 of 98 cases $(38 \%)$ with hearing thresholds $>60 \mathrm{~dB}$ at $4 \mathrm{kHz}$. In our study, eight of 59 bilateral subjects $(13.6 \%)$ experienced hearing loss at this level in at least one ear, which suggests conservatively a prevalence of dead regions in $\sim 5 \%$ of subjects at this frequency and likely an increasing prevalence at higher sound frequencies where hearing loss was deeper. To evaluate a possible influence of dead regions, we contrasted tinnitus spectra when our bilateral cases were divided into subgroups with poor hearing (where dead regions were likely present) and good hearing (where dead regions were less likely to be a factor; see Fig. 3B). The better hearing subgroup gave likeness ratings that increased incrementally up to $10 \mathrm{kHz}$, whereas the poorer hearing group gave likeness ratings that peaked at $6 \mathrm{kHz}$ and declined at higher frequencies. The decline at higher frequencies seen in the poorer hearing group may have occurred because some of the subjects had difficulty hearing these sounds, and the sounds may have been perceived as distorted or noise-like, owing to the presence of cochlear dead regions. This interpretation, if accepted, implies that peaks obtained in tinnitus spectra when hearing function is poor may be spurious and not necessarily present in the tinnitus being measured. It should be noted that tinnitus spectra similar to those of the better hearing subjects in Figure 3B were found in unilateral cases where pitch judgments would have been assisted by the better hearing ear (Fig. 6).

A third factor that may affect the measurement of tinnitus spectra is an impaired ability to discriminate among the sounds that were presented for likeness judgments, owing to impairments in pitch discrimination that have been reported for subjects with hearing loss (Freyman and Nelson 1991). Because the majority of our subjects had hearing loss, it is possible that they judged high-frequency sounds to resemble their tinnitus, in proportion to the degree to which the sounds approached a single pitch that characterized 
the tinnitus percept. While we cannot discount a role for impaired discrimination ability, several observations do not favor this factor as a major determinant of the results. Subjects in our study completed a Familiarization Program that gave them experience with a wide range of sound frequencies up to $12 \mathrm{kHz}$ before tinnitus spectra were measured. This procedure did not specifically test for discrimination ability, but it did give subjects comparative experience with the range of sound frequencies they were later to hear. In agreement with data presented by Freyman and Nelson (1991), frequency discrimination thresholds reported by Noreña et al. (2002) for tinnitus subjects were $<10 \%$ of $\mathrm{CF}$ at $6,500 \mathrm{~Hz}$, which if applicable to our sample, implies that our tinnitus subjects could distinguish the sounds they heard. Cases of tinnitus associated with notched audiograms are also informative. While assessment of tinnitus spectra in such cases has not been systematically explored, one subject in our series and two reported by Noreña et al. (2002) gave likeness ratings that reached asymptote in the notch and diminished outside of it. In these cases, discriminative judgments were made between frequencies below and above the notched region. It is also noteworthy that RI functions appear to track the tinnitus spectrum in notched cases (one subject in the present series; an additional case is reported by Roberts 2007) as well as the tinnitus spectrum associated with presbycusis (the majority of subjects in our series). In determining RI functions, our subjects were asked not to assess the pitch of a sound but to gauge the depth of tinnitus suppression induced by it. The agreement of the two methods suggests that likeness judgments and RI functions reflect fundamental properties of tinnitus and not impairments in discrimination ability.

\section{RI functions}

To our knowledge, RI functions that relate RI depth and duration to the center frequency of masking sounds have not previously been reported in the tinnitus literature. The RI function we observed paralleled the tinnitus spectrum, with RI depth increasing first at the audiometric edge and then continuing to rise with the depth of hearing loss to the limit of hearing. Tinnitus bandwidth also appeared to have an influence. RI depth induced by sounds with CFs in the region of hearing impairment was greater for hissing than for ringing and tonal tinnitus, possibly because the bandwidth of the BPN15 maskers that were used to measure RI were most closely matched to the hissing sensation. RI induced by white noise also pointed to an influence of bandwidth. This stimulus induced greater RI than
BPN15 maskers with CFs $<2 \mathrm{kHz}$, presumably because higher frequencies were contained in the white noise sound. However, BPN15 maskers with CFs in the region of hearing impairment produced greater RI than white noise, possibly because the sound spectrum more closely approximated the spectrum of the tinnitus.

One aspect of our findings that is not well understood is the considerable between-subject variability we observed in RI depth and duration. When our cohort of 59 subjects with bilateral tinnitus was considered, $69.5 \%$ reported at least some degree of RI defined as a suppression of -1.0 or greater ( $20 \%$ of scale) averaged over three trials during RI testing (Fig. 4B). Of the total bilateral sample, $22.0 \%$ reported near elimination of tinnitus to at least one masker averaged over three trials, and $18.6 \%$ reported RI duration persisting between 35 and $45 \mathrm{~s}$ (the latter a time-out limit). Although three subjects reported complete tinnitus suppression for the entire RI test and some subjects anecdotally for longer periods, RI duration measured at its peak was on the order of $25 \mathrm{~s}$ for most subjects (Fig. 4B). The prevalence of RI in our data (some degree of RI reported by $\sim 69.5 \%$ of bilateral cases) is less than the reports by Henry and Meikle (2000) and Vernon and Meikle (2003) that RI can be demonstrated in $\sim 88 \%$ of tinnitus cases. Because RI functions were determined here, one might have expected a higher prevalence of RI in our sample. RI of longer duration has also been reported when measured for its full extent (no timeout), following $60 \mathrm{~s}$ of masking (twice the masker duration we used to measure RI functions). Vernon and Meikle (2003) found that $43 \%$ of patients attending a tinnitus clinic experienced RI lasting over 2 min after a noise masker was presented for $1 \mathrm{~min}$ at a level $10 \mathrm{~dB}$ above a level that masked the tinnitus.

One factor contributing to RI variability in our data was masking level. Masking level (the difference between tinnitus and RI masker loudness, see Fig. 5C) declined with $\mathrm{CF}$, in part because elevated hearing thresholds reduced the sound level that could be delivered within the limit of $95 \mathrm{~dB}$ SPL. When we partitioned our bilateral subjects into subgroups exhibiting good or poor RI, we found that masking levels were on average $12.7 \mathrm{~dB}$ higher for the good RI group at all CFs (Fig. 5C). This finding is consistent with previous reports that RI deepens as the level of the masker rises above the level required to mask the tinnitus (called "minimum masking level" or MML in the literature; see Terry et al. 1983; Tyler et al. 1984, 2000). MML is not identical to masking level as measured here, although the two measures are necessarily correlated when the loudness of RI maskers is manipulated. If it is accepted that masking level is important, it would have been expected that 
partitioning our sample for depth of RI would have selected out this variable. This line of reasoning implies that the dependence of RI on CF evident in Figure 2 may have been more accentuated had it been possible to preserve a more adequate masking level for BPN15 sounds of high CF. Another factor that would have been expected to affect RI is the presence of dead regions that impede the delivery of frequency specific input to the affected tonotopic regions of A1. However, while an important consideration, the presence of dead regions appears unlikely to explain the absence of RI in subjects aged $\leq 50$ years where mean hearing thresholds were found to be in the normal range up to $12 \mathrm{kHz}$. Why our comparatively younger cases were resistant to RI remains to be determined. A contribution from dead regions cannot be ruled out because these have been reported even when hearing thresholds are within the normal range (Weisz et al. 2006). Alternatively, we cannot discount the possibility that the bandwidth match of BPN15 maskers to the tinnitus was especially poor in younger subjects or that these cases represent a distinctive tinnitus type. Study of RI-resistant cases of tinnitus may be a fruitful topic for continued investigation. The RI functions obtained here provide a baseline against which future enhancements of RI can be evaluated.

\section{Mechanism of tinnitus and RI}

A noteworthy feature of our results is that although tinnitus spectra and RI functions exhibited a stepwise increment at the edge of normal hearing, both continued to increase across the range of threshold shift in group-averaged data, reaching a plateau and persisting throughout the region of hearing impairment (Fig. 2B). There was little indication that the tinnitus percept or its suppression during RI were focused at the audiometric edge. When peaks were seen in tinnitus spectra, they appeared at higher frequencies near $6 \mathrm{kHz}$ and appeared to be spurious effects of off-place or low-frequency listening that occurred when hearing thresholds reached about $50 \mathrm{~dB}$ HL (Fig. 2). When hearing loss was minimal (Fig. 3B), or in unilateral cases where sound perception was assisted by the better hearing ear (Fig. 6), tinnitus spectra spanned the region of hearing impairment except at $12 \mathrm{kHz}$, which several subjects could not hear. These results suggest that tinnitus percepts are not confined to sound frequencies that are over-represented at the edge of normal hearing following tonotopic map reorganization (Rauschecker 1999) or to sound frequencies that may be enhanced by contrast mechanisms at the edge of normal hearing (Llinás et al. 2005), although such mechanisms may contribute to the sound of tinnitus. Rather, our results are more consistent with the hypothesis that tinnitus percepts are generated at least in part by synchronous neural activity that develops among neurons that have retained their functional identities in the hearing loss region and are heard in accordance with their location in the cortical place map (Eggermont and Roberts 2004). It should be noted that tinnitus spectra cannot disambiguate contributions arising from synchronous neural activity from those due to changes in the spontaneous firing rate of neurons in tonotopic regions affected by hearing loss. However, increased synchronous activity has been detected in animals exposed to traumatic noise, and unlike changes in spontaneous activity which are not frequency selective, changes in neural synchrony are confined to tonotopic regions affected by hearing loss (Seki and Eggermont 2003; Noreña and Eggermont 2003).

Synchronous neural activity in deafferented cortical regions could in principle be generated by several mechanisms. Eggermont and Roberts (2004) proposed that tonotopic map reorganization occurs when auditory neurons that have lost their thalamocortical input begin to respond to inputs conveyed by lateral connections from their unaffected neighbors in the tonotopic map. Although the tuning shift itself does not represent the tinnitus percept, the shift likely does signal enhanced input via lateral connections to tonotopic regions where tinnitus sounds are generated. Input conveyed by lateral connections (which are known to be highly plastic) could be a driving force for enhanced neural synchrony in the affected cortical regions (Eggermont 2007). One mechanism contributing to synchronous network activity may be homeostatic plasticity, which adjusts neural firing thresholds to preserve spiking rates when other sources of input are lost (Turrigiano 2007). A further contributing factor may be impairments in feed-forward inhibition in cortical regions affected by hearing loss (Cruikshank et al. 2007). In normal auditory cortex, this inhibition (which follows after one synaptic delay) appears to preserve the thalamocortical tuning of auditory neurons such that they respond only to frequencies contained in sound stimuli (normal auditory perception). When this source of tuning is lost, neurons may become more sensitive to input via lateral pathways, which may forge synchronous activity through use-dependent mechanisms. RI, on the other hand, may occur when excitation and inhibition are injected by suprathreshold sounds into the affected region, briefly disrupting the synchronous neural activity responsible for tinnitus. RI could reflect a temporary adaptation of neurons involved 
in synchronous activity, a rebalancing of the influence of thalamocortical and lateral synapses on auditory neurons by the masker or other mechanisms that subside with a time constant on the order of 30 s. Although this account of tinnitus and RI emphasizes the role of $\mathrm{A} 1$ as the site of functional changes, effects occurring here could modulate activity in other brain regions that have been implicated in tinnitus by functional imaging studies (Melcher et al. 2000; Lockwood et al. 2001; Leaver et al. 2006; Wienbruch et al. 2006; Smits et al. 2007; Schlee et al. 2007; Weisz et al. 2007).

In agreement with deafferentation models of tinnitus (Eggermont and Roberts 2004; Llinás et al. 2005; Rauschecker 1999), several aspects of our findings implicated hearing impairment as a factor. In particular, (1) tinnitus spectra and RI functions increased incrementally at the edge of normal hearing and proportionately with the extent of threshold shift, (2) the tinnitus spectrum shifted to higher frequencies with increases in the audiometric edge, and (3) the ear with poorer hearing was a predictor of the tinnitus ear in unilateral cases. In addition, when compared to age-matched controls without tinnitus, tinnitus subjects exhibited hearing thresholds that were elevated on average by $\sim 12 \mathrm{~dB}$ between $2-10 \mathrm{kHz}$ for subjects $>50$ years old and by the same amount between $2-4 \mathrm{kHz}$ for younger subjects $\leq 50$ years old. Hence, we detected a small hearing impairment in our younger group even though their mean thresholds were within the audiologically normal range $(<25 \mathrm{~dB}$ HL). Nonetheless, our older control subjects showed large threshold shifts outside of the normal range at frequencies above $8 \mathrm{kHz}$ but did not report tinnitus, whereas our younger subjects with better hearing thresholds in this frequency range experienced tinnitus. Elevation of the hearing threshold, while clearly correlated with tinnitus, was not sufficient for its occurrence.

One factor that may potentially discriminate between subjects with and without tinnitus is the prevalence of cochlear dead regions in the two groups regardless of age. It is also possible that many of our older control subjects showing highfrequency hearing loss without tinnitus may eventually come to experience tinnitus, which would reduce the disparity between the presence or absence of hearing loss and of tinnitus. Ling et al. (2005) and Caspary et al. (2005) have documented age-related changes in GABAergic and glycinergic inhibition in central auditory structures that may account for an increase in the incidence of tinnitus with aging. Nevertheless, what protects some elderly individuals with hearing loss from tinnitus is at present undetermined.

\section{ACKNOWLEDGMENT}

This research was funded by the Canadian Institutes of Health Research (CIHR NET Programme), NSERC of Canada, and the American Tinnitus Association. Dave Thompson, Oksana Smyczyk, Phillip Gander, and Stephanie Thai assisted in conducting the study. We thank two anonymous reviewers and Richard Tyler for their insightful comments on an earlier version of the manuscript.

\section{REFERENCES}

Aazh H, Moore BCJ. Dead regions in the cochlea at $4 \mathrm{kHz}$ in elderly adults: relation to absolute threshold, steepness of audiogram, and pure-tone average. J. Am. Acad. Audiol. 18:97-106, 2007.

Borg G, Borg E. A new generation of scaling methods: level anchored ratio scaling. Psychologica 28:15-45, 2001.

Caspary DM, Schatteman TA, Hughes LF. Age-related changes in the inhibitory response properties of dorsal cochlear nucleus output neurons: role of inhibitory inputs. J. Neurosci. 25:10952-10959, 2005.

Cruikshank SJ, Lewis TJ, Connors BW. Synaptic basis for intense thalamocortical activation of feedforward inhibitory cells in neocortex. Nat. Neurosci. 10:462-468, 2007.

Eggermont JJ. Correlated neural activity as the driving force for functional changes in auditory cortex. Hear. Res. 229:69-80, 2007.

Eggermont JJ, Komiya H. Moderate noise trauma in juvenile cats results in profound cortical topographic map changes in adulthood. Hear. Res. 142:89-101, 2000.

Eggermont JJ, Roberts LE. The neuroscience of tinnitus. Trends Neurosci. 27:676-682, 2004.

Feldman H. Homolateral and contralateral masking of tinnitus by noise-bands and by pure tones. Audiology 10:138-144, 1971.

Florentine M, Houstsma AJM. Tuning curves and pitch matches in a listener with a unilateral, low frequency hearing loss. J. Acoust. Soc. Am. 73:961-965, 1983.

Freyman RL, NeLson DA. Frequency discrimination as a function of signal frequency and level in normal-hearing and hearingimpaired listeners. J. Speech Hear. Res. 34:1371-1386, 1991.

Han LA, Poulsen T. Equivalent threshold sound pressure levels for Sennheiser HDA 200 earphone and etymotic research ER-2 insert earphone in the frequency range $125 \mathrm{~Hz}$ to $16 \mathrm{kHz}$. Scand. Audiol. 27:105-112, 1998.

Harrison RV, Nagasawa A, Smith DW, Stanton S, Mount RJ. Reorganization of auditory cortex after neonatal high frequency cochlear hearing loss. Hear. Res. 54:11-19, 1991.

Henry JA, Meikle MB. Psychoacoustic measures of tinnitus. J. Am. Acad. Audiol. 11:138-155, 2000.

Huss M, Moore BCJ. Dead regions and pitch perception. J. Acoust. Soc. Am. 117:3841-3852, 2005a.

Huss M, Moore BCJ. Dead regions and noisiness of pure tones. Int. J. Audiol. 44:599-611, 2005b.

KIAng NYS, Moxon FC, Levine RA. Auditory nerve activity in cats with normal and abnormal cochleas. In: Wolstenholme GEW, Knight J (eds) Ciba Foundation Symposium On Sensorineural Hearing Loss. London, UK, Churchill, pp. 241-273, 1969.

Kuk FK, Tyler RS, Russell D, Jordan H. The psychometric properties of a tinnitus handicap questionnaire. Ear Hear. 11:434-445, 1990.

Leaver A, Renier L, Purcell J, Costanzo M, Fieger A, Morgan S, Kim HJ, Rauschecker JP. Auditory cortical map plasticity in tinnitus. Soc. Neurosci. Abstract 800.2/E29, 2006. 
Ling LL, Hughes LF, Caspary DM. Age-related loss of the GABA synthetic enzyme glutamic acid decarboxylase in rat primary auditory cortex. Neuroscience 132:1103-1113, 2005.

Llinás R, Urbano FJ, Leznik E, Ramírez RR, van Marle HJ. Rhythmic and dysrhythmic thalamocortical dynamics: GABA systems and the edge effect. Trends Neurosci. 28:325-333, 2005.

Lockwood AH, Wack DS, Burkard RF, Coad ML, Reyes SA, Arnold SA, SALVI RJ. The functional anatomy of gaze-evoked tinnitus and sustained lateral gaze. Neurology. 56:472-480, 2001.

Melcher JR, Sigalovsky IS, Guinan JJ, LeVine RA. Lateralized tinnitus studied with functional magnetic resonance imaging: abnormal inferior colliculus activation. J. Neurophysiol. 83:1058-1072, 2000.

Mrtchell C. The masking of tinnitus with pure tones. Audiology 22:73-87, 1983.

NoReÑa AJ, EgGERMONT JJ. Changes in spontaneous neural activity immediately after an acoustic trauma: implications for neural correlates of tinnitus. Hear. Res. 183:137-153, 2003.

Noreña A, Micheyl C, Cherz-Croze S, Collet L. Psychoacoustic characterization of the tinnitus spectrum: implications for the underlying mechanisms of tinnitus. Audiol. Neurootol. 7:358$369,2002$.

Rajan R, Irvine DR, Wise LZ, Heil P. Effect of unilateral partial cochlear lesions in adult cats on the representation of lesioned and unlesioned cochleas in primary auditory cortex. J. Comp. Neurol. 338:17-49, 1993.

RAUSCHECKER JP. Auditory cortical plasticity: a comparison with other sensory systems. Trends Neurosci. 22:74-80, 1999.

RoBerts LE. Residual inhibition. Prog. Brain Res. 166:487-495, 2007.

Roberts LE, Moffat G, Bosnyak DJ. Residual inhibition functions in relation to tinnitus spectra and auditory threshold shift. Acta Otolaryngol. Suppl. 556:27-33, 2006.

Robertson D, IRvine DRF. Plasticity of frequency organization in auditory cortex of guinea pigs with partial unilateral deafness. J. Comp. Neurol. 282:456-461, 1989.

Schlee W, Weisz $\mathrm{N}$, Dohrmann $\mathrm{K}$, Hartmann $\mathrm{T}$, Elbert $\mathrm{T}$. Unravelling the tinnitus distress network using single trial auditory steady-state responses. In: Cheyne D, Ross B, Stroink G, Weinberg H (eds) New Frontiers in Biomagnetism. Proceedings of the 15th International Conference on Biomagnetism Amsterdam, Elsevier, International Congress Series, vol. 1300, pp. 73-76, 2007.

Seki S, EgGermont JJ. Changes in spontaneous firing rate and neural synchrony in cat primary auditory cortex after localized toneinduced hearing loss. Hear. Res. 180:28-38, 2003.

Smits M, Kovacs S, de Ridder D, Peeters RR, van Hecke P, Sunaert S. Lateralization of functional magnetic resonance imaging (fMRI) activation in the auditory pathway of patients with lateralized tinnitus. Neuroradiology 49:669-679, 2007.

Terry AMP, Jones DM, Davis BR, Slater R. Parametric studies of tinnitus masking and residual inhibition. Br. J. Audiol. 17:245256, 1983.

TuRrigiano G. Homeostatic signaling: the positive side of negative feedback. Curr. Opin. Neurobiol. 17:318-324, 2007.

TyLer RS. Psychoacoustical measurement. In: Tyler RS (ed) Tinnitus Handbook. San Diego, CA, Singular Publishing Group, pp. 149 $180,2000$.

Tyler RS, Conrad-Ames D. Masking of tinnitus compared to masking of pure tones. J. Speech Hear. Res. 27:106-111, 1984.

Tyler RS, Conrad-Ames D, Smith P. Post-masking effects of sensorineural tinnitus: a preliminary investigation. J. Speech Hear. Res. 27:466-474, 1984

Vernon JA, MeIKLE MB. Tinnitus: clinical measurement. Otolaryngol. Clin. N. Am. 36:293-305, 2003.

Weisz N, Hartmann T, Dohrmann K, Schlee W, Norena A. Highfrequency tinnitus without hearing loss does not mean absence of deafferentation. Hear. Res. 222:108-114, 2006.

Weisz N, Müller S, Schlee W, Dohrmann K, Hartmann T, Elbert T. The neural code of auditory phantom perception. J. Neurosci. 27:1479-1484, 2007.

Wienbruch C, Paul I, Weisz N, Elbert T, Roberts LE. Frequency organization of the $40-\mathrm{Hz}$ auditory steady-state response in normal hearing and in tinnitus. Neuroimage 3:80-194, 2006. 